\title{
Employee substitutability as a tool to improve the robustness in personnel scheduling
}

\author{
Jonas Ingels $^{1} \cdot$ Broos Maenhout $^{1}$
}

Received: 7 July 2015 / Accepted: 18 February 2017

(C) Springer-Verlag Berlin Heidelberg 2017

\begin{abstract}
Organisations usually construct personnel rosters under the assumption of a deterministic operating environment. In the short term, however, organisations operate in a stochastic environment as operational variability arises. This variability leads to the occurrence of unexpected events such as employee absenteeism and/or a demand for personnel that is higher or lower than expected. In order to deal with these uncertainties, organisations need to adopt proactive and reactive scheduling strategies to protect the personnel roster and to respond to this operational variability, respectively. In this paper, we discuss a proactive approach that exploits the concept of employee substitutability to improve the flexibility of a personnel shift roster to respond to schedule disruptions. We propose a pre-emptive programming approach to construct a medium-term personnel shift roster that maximises the employee substitutability value. Moreover, we assess different proactive strategies to introduce robustness with respect to the definition and formulation of employee substitutability and different reactive strategies that impact the decision freedom for schedule recovery. The robustness of the generated personnel shift rosters is evaluated using a three-step methodology of roster construction, daily simulation and optimisation, and evaluation.
\end{abstract}

Keywords Personnel scheduling · Robustness · Employee substitutability · Pre-emptive programming

$\varangle$ Jonas Ingels

Jonas.Ingels@UGent.be

1 Faculty of Economics and Business Administration, Ghent University, Tweekerkenstraat 2, 9000 Ghent, Belgium 


\section{Introduction}

Organisations often face a degree of uncertainty during their operations. Van den Bergh et al. (2013) distinguish three sources of operational variability, i.e. uncertainty of demand, uncertainty of capacity and uncertainty of arrival. This operational variability may disrupt or have a negative impact on the execution of the operations of an organisation. As a result, the impact of uncertainty needs to be considered in order to attain the desired service level in such a way that the associated costs are minimised and the employee satisfaction is optimised. Personnel costs significantly contribute to the operating costs of organisations (Ernst et al. 2004b; Van den Bergh et al. 2013) and employee satisfaction is an important objective in personnel scheduling (Bard and Purnomo 2005; Pato and Moz 2008; Topaloglu and Selim 2010). As such, the personnel planner should account for uncertainty in the personnel planning process. This process generally consists of three hierarchical phases where the higher phases constrain the lower phases (Abernathy et al. 1973; Burke et al. 2004). We distinguish the strategic staffing phase, the tactical scheduling phase and the operational allocation phase. Each phase is characterised by a certain level of decision freedom and uncertainty.

In the staffing phase, long-term capacity decisions that involve the required personnel mix, i.e. the personnel competencies and the personnel budget, are made to meet the (aggregated) service demand. During the scheduling phase, a personnel roster is constructed for a medium-term period by assigning the available personnel resources to specific duties, i.e. working assignments. Given the longer-term nature of these strategic and tactical decisions, the personnel planner is confronted with a certain degree of uncertainty which decreases for lower phases in the hierarchical planning process. As such, these strategic and tactical decisions are made based on a number of assumptions about the future service demand and employee availability. Only in the short-term operational allocation phase, when the roster is executed, up-to-date information about the operating environment is available. In this respect, the personnel planner receives recent and accurate information about the service demand and employee availability. Due to operational variability, this information may differ from the previously made assumptions and disrupt the personnel roster. This uncertainty may force the personnel planner to adjust the original personnel roster in the short term.

In order to manage the impact of this operational variability, a proactive and/or reactive mechanism may be introduced. A proactive mechanism tries to construct a personnel roster in the tactical scheduling phase that is able to absorb unexpected events (Dück et al. 2012) or to improve the adjustment capability (Ionescu and Kliewer 2011). A reactive mechanism determines the required operational adjustments to the personnel roster to efficiently and effectively restore its workability in response to schedule disruptions. Since hedging against all unexpected events in a proactive way would be very expensive, such a reactive mechanism is indispensable to deal with schedule disruptions. However, reactive changes in response to schedule disruptions are typically limited by feasibility rules, may be very costly and may be at the expense of the personnel. It is, therefore, important to construct robust personnel rosters by considering specific proactive mechanisms that ensure the flexibility of a personnel 
roster, i.e. the availability of those adjustment possibilities that enable the personnel planner to efficiently recover the personnel roster with a small number of adjustments and a minimal impact on the service level, the personnel costs and the personnel satisfaction.

In this paper, we focus on the personnel shift scheduling problem, which encompasses the assignment of a multi-skilled workforce to cover the shift demand for a medium-term period. We investigate how the short-term adjustment capability or flexibility embedded in the personnel roster may be improved proactively by maximising the employee substitutability value. In general, a substitution exists if an employee can take over the skill-shift assignment of another employee on a particular day. In this respect, we discuss three mechanisms to perform a personnel substitution, i.e. a between-skill substitution, a within-skill substitution and a day-off-to-work substitution. We consider and analyse different ways to define, model and measure employee substitutability. As such, we define employee substitutability on the level of an individual employee and on the group level where the degree of cross-training of the workforce assigned to a particular shift is considered. Moreover, we propose and compare different strategies to model employee substitutability and to measure the number of substitution possibilities and the corresponding employee substitutability value.

We provide guidelines to define the tactical decision process as a better proxy for the short-term operating environment by considering the operational variability. Therefore, we validate the improved roster robustness that is achieved by maximising the employee substitutability value with a three-step methodology. In the first step, a personnel shift roster is constructed by applying a two-phase pre-emptive programming approach to evaluate the trade-off between robustness and the cost of robustness. In the first phase, we construct a personnel shift roster solely considering the objective of cost minimisation. In the second phase, a proactive strategy is applied to hedge against uncertainty as we maximise the employee substitutability value of the personnel shift roster given a restriction on the allowable cost increase on top of the minimum cost corresponding to the minimum cost personnel shift roster. The second step imitates the operational allocation phase for each day of the planning horizon and alternates between a simulation step and a reactive roster adjustment step. In the simulation step, the uncertainty of demand and uncertainty of capacity is imitated, which may lead to schedule disruptions. The reactive adjustment mechanism tries to solve these disruptions and balances supply and demand by allowing certain types of personnel substitutions. In a third step, the robustness of the original personnel shift roster is evaluated based on the planned and expected actual performance. The second and third steps are repeated multiple times to obtain a clear idea of the obtained robustness. Computational experiments are conducted both in a real-life and a full-factorial artificial setting to investigate the impact of different demand profiles.

The remainder of this paper is organised as follows. We give an overview of the relevant literature in Sect. 2. In Sect. 3, we define the three-step methodology, which includes the tactical scheduling phase, the operational allocation phase and the robustness evaluation. We describe different types of employee substitutions and formulate the personnel shift scheduling problem to maximise the employee substitutability value on an individual and group level. In Sect. 4, we define and explain the pre- 
emptive two-phase approach to construct a medium-term personnel shift roster. The test design, test instances and computational experiments are discussed in Sect. 5. We provide conclusions in Sect. 6.

\section{Literature overview}

The personnel management and planning process is extensively investigated in the operations research literature (Burke et al. 2004; Bruecker et al. 2015; Dorne 2008; Ernst et al. 2004a, b; Van den Bergh et al. 2013). These review papers discuss the personnel shift scheduling problem as one of the most important problems in personnel scheduling. There are many different application areas for the personnel shift scheduling problem such as in manufacturing, airline industry, call centres, healthcare, transportation, etc. Given the multitude of application areas, problem types, forms and formulations and the complex nature of the shift scheduling problem, many exact and heuristic solution methodologies have been proposed in literature to construct personnel shift rosters (Burke et al. 2004; Ernst et al. 2004a, b; Van den Bergh et al. 2013). These solution methodologies often utilise some type of decomposition to reduce the size and complexity of the problem (Van den Bergh et al. 2013). In this respect, a complex problem can be partitioned into a series of smaller and more manageable (sub)problems or phases. These phases solve distinct parts of the original problem and differ in the constraints and/or objectives they consider. Decomposition is applied in solution methodologies such as pre-emptive programming (Topaloglu and Ozkarahan 2004), branch-and-bound (Trivedi and Warner 1976), column generation (Bard and Purnomo 2005) and branch-and-price (Ingels and Maenhout 2015; Maenhout and Vanhoucke 2010).

The construction of robust personnel rosters is a topic that has received only limited attention in the literature. Given the occurrence of uncertainty in the operational allocation phase, organisations need to apply proactive and reactive strategies to deal with the actual demand and employee availability. A proactive approach builds in a certain degree of robustness in the original roster. This built-in robustness improves both the absorption and adjustment capability of the original roster during the operational allocation phase when unexpected events occur. Thus, according to the literature, robustness involves both stability (Dück et al. 2012) and flexibility (Ionescu and Kliewer 2011) to ensure a stable and high-quality personnel roster (Gross et al. 2017). Dück et al. (2012) define a stable roster as a roster that has a high absorption capability and consequently exhibits a small number of adjustments when the operating environment changes. According to Ionescu and Kliewer (2011), a roster is flexible or has a high adjustment capability when there are enough possibilities for schedule changes to efficiently recover from unexpected events. Note that strategies promoting stability have a different focus than strategies promoting flexibility. Both types of strategies can complement each other to increase the personnel roster robustness. In case the inherent flexibility in a roster is small, strategies that promote stability may be introduced to increase the robustness and vice versa.

Different strategies are mentioned in the literature to increase the robustness by focusing on the stability of a personnel roster. A common approach is to include 
buffers such as time buffers or capacity buffers. Time buffers can be used to deal with uncertain activity durations in project management (uncertainty of arrival) (Hazir et al. 2010), unexpected delays of tasks in personnel task scheduling problems (uncertainty of arrival) (Dück et al. 2012; Ehrgott and Ryan 2002; Tam et al. 2011) and machine breakdowns in job shop scheduling (uncertainty of capacity) (Davenport et al. 2001). Capacity buffers occur under different names, among which reserve crew and preferred requirements. The introduction of reserve duties as a means to improve robustness has been widely studied in the airline industry given that unforeseen demand (uncertainty of demand) (Dillon and Kontogiorgis 1999), employee sickness (uncertainty of capacity) (Dillon and Kontogiorgis 1999; Moudani and Mora-Camino 2010), technical failures (uncertainty of capacity) (Rosenberger et al. 2002; Sohoni et al. 2006) and unexpected task delays due to adverse weather conditions or unscheduled maintenance (uncertainty of arrival) (Sohoni et al. 2006) may lead to severe and costly delays. Similarly, reserve duties are investigated in the context of the railway crew rescheduling problem as a reaction to uncertainty of infrastructure capacity (Potthoff et al. 2010) and in the context of the personnel shift scheduling problem to deal with uncertainty of demand and capacity (Ingels and Maenhout 2015). Ingels and Maenhout (2015) consider a personnel shift scheduling problem with a homogeneous workforce and aim to improve the stability of a roster by introducing reserve duties. The definition of preferred staffing requirements is another way to install staff buffers. These requirements are higher than the minimum staffing requirements and are typically applied in hospitals (De Causmaecker and Vanden Berghe 2003; Dowsland and Thompson 2000; Topaloglu and Selim 2010). Moreover, apart from time or resource buffers, it is also possible to improve the robustness by focusing on the teams that perform a sequence of tasks. Tam et al. (2014) study the airline crew scheduling problem and define the concept of unit crewing as keeping crew with different skills and ranks together for as long as possible in a pairing to minimise delay propagations due to uncertainty of arrival in the operational phase.

In contrast, the number of substitution possibilities or swaps is a main indicator for the robustness in crew and aircraft scheduling (Dück et al. 2012) because it is an indication of the roster flexibility. Indeed, a crew and/or aircraft swap is one of the available recovery actions to overcome operational disruptions due to uncertainty of arrival (Abdelghany et al. 2004, 2008; Eggenberg et al. 2010; Gao et al. 2009; Ionescu and Kliewer 2011) and uncertainty of capacity (Abdelghany et al. 2004). Shebalov and Klabjan (2006) focus on the maximisation of move-up crews. Moveup crews are crews that can be swapped to overcome operational disruptions such as airport shutdowns creating uncertainty of arrival. A crew swap is recognised as a cost-efficient option when disruptions occur. The authors build robust rosters using a two-phase approach by first minimising the crew costs and in a second phase the number of move-up crews is maximised within the limits of an allowable cost increase. The proposed crew rosters are evaluated by generating random disruptions that are solved utilising a recovery module. Ionescu and Kliewer (2011) also introduce swaps in the scheduling phase to improve the flexibility of the original roster to respond to delays caused by weather and aircraft breakdowns (uncertainty of arrival). The authors propose a stochastic optimisation model for which the recourse function represents the benefits of swaps. In contrast to Shebalov and Klabjan (2006), the focus is more 
on the utility of a swap in the time-space network rather than a pure maximisation of the number of swaps. This utility depends on the likelihood of delay propagations. The authors evaluate the roster robustness through a scenario-based simulation model and test their approach on real-world test instances. Substitution possibilities can also be obtained through cross-training. Campbell (1999) investigates the impact of the cross-utilisation of employees for different levels of demand variability (uncertainty of demand) and employee cross-training. Similarly, Olivella and Nembhard (2016) determine the optimal level of cross-training in work teams to deal with variations in the demand mix and employee availability.

\section{Problem definition, formulation and methodology}

We investigate how the flexibility of a personnel shift roster may be increased as a result of improving the employee substitutability of a heterogeneous multi-skilled workforce in order to recover efficiently from schedule disruptions. In this study, we extend the idea of swaps in a personnel shift scheduling context. Ionescu and Kliewer (2011) and Shebalov and Klabjan (2006) study the concept of swaps in an airline scheduling context as a possible reaction to delays due to arrival uncertainty. A swap is a two-direction mechanism that exchanges duties between employees, i.e. employees that have a later assignment are assigned to an earlier assignment, while the employees from a disrupted assignment are assigned to the later assignment. In airline scheduling, duties may have different start times and durations, which are subject to operational variability. Therefore, it can be useful to swap duties between employees. In the context of a shift scheduling problem, this practice is often not useful because the assigned duties have fixed start and end times. An unexpected increase in demand during a particular shift, for example, does not benefit from a swap between employees because this would not resolve the understaffing. In this case, an employee needs to be reassigned from an overstaffed shift or from a day off to the understaffed shift, i.e. a substitution needs to be performed.

Therefore, we study how and to which extent employee substitutions can serve as a proactive methodology to improve the robustness of a personnel shift roster. In this respect, we maximise the employee substitutability value to add flexibility to the decision-making process in the operational allocation phase. The personnel scheduling problem under study, which aims to create an increased flexibility by including employee substitutability in the objective function, is relevant for many application areas to facilitate the recovery from disruptions. An important application lies in employee self-scheduling (Bailyn et al. 2007) where the management typically first proposes a feasible (minimum cost) roster indicating the line-of-work for each individual employee. Subsequently, employees are able to adapt their own schedule. Bailyn et al. (2007), however, identified that employees are not able to adapt their own schedule taking the stipulated (time-related) rules and regulations into account. In this way, self-scheduling may lead to conflicts or even infeasible schedules that need to be resolved by the employees or the management. The resolution of these conflicts can be executed more effectively and efficiently if employee substitutability is proactively considered when the personnel roster is constructed. 
In this paper, we utilise a three-step methodology to validate the impact of employee substitutability on the robustness of a personnel shift roster, i.e.

- In the first step, i.e. the (tactical) scheduling phase, a baseline personnel shift roster is constructed for a medium-term period based upon a proactive strategy. A new objective is included to optimise the employee substitutability. We provide the description and model formulation of the problem under study in Sect. 3.1.

- In the second step, we start from the baseline personnel shift roster and imitate the operational allocation phase for each day of the planning horizon. This operational phase consists of a simulation component and a reactive adjustment component. Each day we simulate the uncertainty of demand and the uncertainty of capacity. This simulated operational variability may lead to a different actual demand for staff and unexpected employee absenteeism. In order to solve these schedule disruptions, the personnel roster is adapted by performing certain reassignments or personnel substitutions on the day under consideration. Since the planning horizon is small, the adjustment capability heavily depends on the built-in robustness or flexibility, i.e. the employee substitutability. We describe both components that constitute the operational allocation phase in Sect. 3.2.

- In the third step, we evaluate the robustness of the baseline personnel shift roster through a comparison of the planned and expected actual performance of the roster (Sect. 3.3).

The general methodology of validating the proactively obtained robustness by imitating the reactive operational phase is similar to the approach of Abdelghany et al. (2008), Bard and Purnomo (2005) and Ingels and Maenhout (2015). In the remainder of this paper, a personnel shift roster refers to the set of employee schedules for the complete workforce and an employee schedule represents the line-of-work of an individual employee. This line-of-work indicates for each day whether the employee has a day off or works a particular duty, i.e. a working assignment characterised by the corresponding (skill, day, shift)-combination.

\subsection{Tactical scheduling phase}

We study a general tactical shift scheduling problem that assigns employees to shifts during the scheduling phase. The shift assignments cover multiple categorical skills, which means that employees are fully capable of executing a duty in correspondence with their skills and the individual skills cannot be hierarchically ranked (Bruecker et al. 2015). The personnel characteristics and shift characteristics are common in personnel scheduling literature (Burke et al. 2004; Van den Bergh et al. 2013) and the model can be categorised as $A S 2|R V| S|| L R G$ according to the classification of De Causmaecker and Vanden Berghe (2011).

In this phase, we introduce the concept of employee substitutability on the level of an individual employee (Sect. 3.1.1) and on a group level (Sect. 3.1.2). Individual employee substitutability depends on the value and number of substitution possibilities, while group employee substitutability depends on the degree of cross-training of the assigned workforce. Both problems are modelled with a multi-criteria objective function, simultaneously optimising the personnel assignment costs (i.e. the wage 
cost and preference penalty cost), the understaffing cost and the individual or group employee substitutability value. We utilise the following mathematical notation to include employee substitutability in personnel shift rosters, i.e.

\section{Notation}

Sets

$G \quad$ set of skills (index $m$ )

$N \quad$ set of employees (index $i$ )

$D \quad$ set of days (index $d$ )

$S \quad$ set of shifts (index $j$ )

$T_{d j}^{\prime}$

$T_{d j}^{\prime \prime}$

set of shifts that cannot be assigned the day before day $d$ and shift assignment $j$ (index $s$ )

Parameters

\section{$b_{i m}$ \\ $R_{m d j}^{w}$ \\ $R_{m d j}^{w,+}$ \\ $c_{i m d j}^{w}$ \\ $c_{m d j}^{w u}$ \\ $c_{m d j}^{w u,+}$ \\ $p_{i d j}$ \\ $\gamma_{m d j}$ \\ $l_{j}$ \\ $\eta_{i}^{w, \min }$ \\ $\eta_{i}^{w, \max }$ \\ $\theta_{i}^{w, \max }$ \\ $\epsilon$}

$\beta_{m d j}$

Variables

$x_{i m d j}^{w}$

$x_{i d}^{v}$

$x_{m d j}^{w u}$

$x_{m d j}^{w u,+}$

zimdj

set of shifts that cannot be assigned the day after day $d$ and shift assignment $j$ (index $s$ )

1 if employee $i$ possesses skill $m, 0$ otherwise

minimum staffing requirements for shift $j$, day $d$ and skill $m$

additional group staffing requirements for shift $j$, day $d$ and skill $m$

wage cost of assigning an employee $i$ to shift $j$, day $d$ and skill $m$

shortage cost of the minimum staffing requirements for shift $j$, day $d$ and skill $m$

shortage cost of the group staffing requirements for shift $j$, day $d$ and skill $m$

preference penalty cost if an employee $i$ receives a shift assignment $j$ on day $d$

the benefit value of a substitution possibility for shift $j$, day $d$ and skill $m$

duration of shift $j$

minimum number of hours that need to be assigned to employee $i$ maximum number of hours that can be assigned to employee $i$ maximum number of consecutive working assignments for employee $i$ maximum number of substitution possibilities an employee can offer on a day

maximum number of substitution possibilities for shift $j$, day $d$ and skill $m$

1 if employee $i$ receives a shift assignment $j$ for skill $m$ on day $d, 0$ otherwise

1 if employee $i$ receives a day off on day $d, 0$ otherwise the shortage of employees for shift $j$, day $d$ and skill $m$ [w.r.t. the minimum staffing requirements (Eq. 2)] the shortage of employees for shift $j$, day $d$ and skill $m$ [w.r.t. the group staffing requirements (Eq. 14)] 1 if a substitution possibility exists for employee $i$ for shift $j$, day $d$ and skill $m, 0$ otherwise 


\subsubsection{Individual employee substitutability}

The consideration of employee substitutability on an individual employee level during the construction of a personnel shift roster entails a maximisation of the weighted sum of the value of substitution possibilities between employees. This means that not only the number of substitution possibilities is taken into account but also the weight of the specific (skill, day, shift)-combinations in the personnel shift roster. Hence, we aim to optimise the number and position of the introduced substitution possibilities for a particular skill.

A substitution is defined as the possibility of an employee to take over the assignment of another employee on the same day. We distinguish three different types of substitutions, which are illustrated in Fig. 1. These types are defined as follows:

(a) A between-skill substitution indicates that a working employee can be reassigned to another skill during the same or another shift on the same day (Fig. 1a). (b) A within-skill substitution is the potential reassignment of an employee from one shift to another shift within the same skill category (Fig. 1b).

(c) A day-off-to-work substitution consists of the potential reassignment of an employee with a day off to a working shift subject to his/her competencies (Fig. 1c).

These substitution types are taken into account in the following mathematical model, i.e.

$$
\begin{aligned}
\min & \sum_{i \in N} \sum_{m \in G} \sum_{d \in D} \sum_{j \in S}\left(c_{i m d j}^{w}+p_{i d j}\right) x_{i m d j}^{w}+\sum_{m \in G} \sum_{d \in D} \sum_{j \in S} c_{m d j}^{w u} x_{m d j}^{w u} \\
& +\sum_{i \in N} \sum_{m \in G} \sum_{d \in D} \sum_{j \in S} \gamma_{m d j}\left(1-z_{i m d j}\right)
\end{aligned}
$$

In this study, we optimise the personnel assignment costs and the understaffing of the minimum staffing requirements (Eq. 1a). Simultaneously, we maximise the employee

\begin{tabular}{|c|c|c|c|c|c|c|c|}
\hline & \multicolumn{2}{|c|}{ Shift 1} & \multicolumn{2}{|c|}{ Shift 2} & \multicolumn{2}{|c|}{ Shift 3} & \multirow{2}{*}{$\begin{array}{l}\text { Day } \\
\text { off }\end{array}$} \\
\hline & Skill 1 & Skill 2 & Skill 1 & Skill 2 & Skill 1 & Skill 2 & \\
\hline E1 & 0 & 0 & ${ }^{1} k$ & 0 & 0 & 0 & 0 \\
\hline E2 & 0 & 0 & 0 & $\Delta_{1}$ & 0 & 0 & 0 \\
\hline
\end{tabular}
substitutability value on an individual employee level (Eq. (1b)). The personnel assign-

\begin{tabular}{|c|c|c|c|c|c|c|c|}
\hline & \multicolumn{2}{|c|}{ Shift 1} & \multicolumn{2}{|c|}{ Shift 2} & \multicolumn{2}{|c|}{ Shift 3} & \multirow{2}{*}{$\begin{array}{c}\text { Day } \\
\text { off }\end{array}$} \\
\hline & Skill 1 & Skill 2 & Skill 1 & Skill 2 & Skill 1 & Skill 2 & \\
\hline E1 & 0 & 0 & $\rightarrow 1$ & 0 & 0 & 0 & 0 \\
\hline E2 & 14 & 0 & 0 & 0 & 0 & 0 & 0 \\
\hline
\end{tabular}

a

\begin{tabular}{|c|c|c|c|c|c|c|c|}
\hline & \multicolumn{2}{|c|}{ Shift 1} & \multicolumn{2}{|c|}{ Shift 2} & \multicolumn{2}{|c|}{ Shift 3} & \multirow{2}{*}{$\begin{array}{c}\text { Day } \\
\text { off }\end{array}$} \\
\hline & Skill 1 & Skill 2 & Skill 1 & Skill 2 & Skill 1 & Skill 2 & \\
\hline E1 & 0 & 0 & $1 \leftarrow$ & 0 & 0 & 0 & 0 \\
\hline E2 & 0 & 0 & 0 & 0 & 0 & 0 & -1 \\
\hline
\end{tabular}

b

C

Fig. 1 Substitution types. a Between-skill substitutions, b within-skill substitutions, c day-off-to-work substitutions 
ment costs include the wage cost $\left(c_{i m d j}^{w}\right)$ and preference penalty cost $\left(p_{i d j}\right)$ associated with the assignment of an employee to a duty. Note that a lower preference penalty cost indicates a higher willingness to be assigned to that shift (Bard and Purnomo 2005; Maenhout and Vanhoucke 2010) and that the objective function implicitly optimises overstaffing as a cost is incurred for each duty.

$$
\sum_{i \in N} b_{i m} x_{i m d j}^{w}+x_{m d j}^{w u} \geq R_{m d j}^{w} \quad \forall m \in G, \forall d \in D, \forall j \in S
$$

The staffing requirements in Eq. (2) define the number of employees that are required to meet the demand for every skill category, day and shift. This constraint is relaxed by allowing understaffing.

$$
\begin{array}{cc}
\sum_{m \in G} \sum_{j \in S} x_{i m d j}^{w}+x_{i d}^{v}=1 \quad \forall i \in N, \forall d \in D \\
\sum_{m \in G} x_{i m d j}^{w}+\sum_{m \in G} \sum_{S \in T_{d j}^{\prime \prime}} x_{i m(d+1) s}^{w} \leq 1 \quad \forall i \in N, \forall d \in D, \forall j \in S
\end{array}
$$

Equation (3) postulates that an employee should be assigned to either a duty or a day off. The minimum rest period between consecutive shift assignments is ensured by constraint (4).

$$
\begin{array}{cl}
\sum_{m \in G} \sum_{d \in D} \sum_{j \in S} l_{j} x_{i m d j}^{w} \leq \eta_{i}^{w, \max } & \forall i \in N \\
\sum_{m \in G} \sum_{d \in D} \sum_{j \in S} l_{j} x_{i m d j}^{w} \geq \eta_{i}^{w, \min } & \forall i \in N \\
\sum_{d^{\prime}=d}^{d+\theta_{i}^{w, \max }}\left(1-x_{i d^{\prime}}^{v}\right) \leq \theta_{i}^{w, \max } & \forall i \in N, \forall d \in D
\end{array}
$$

The other time-related constraints include the maximum (Eq. 5) and minimum (Eq. 6) number of hours for every employee. Furthermore, the number of consecutive working assignments is limited by Eq. (7).

$$
x_{i m d j}^{w}+z_{i m d j} \leq b_{i m} \quad \forall i \in N, \forall m \in G, \forall d \in D, \forall j \in S
$$

Equation (8) ensures that a substitution possibility only exists if the employee possesses the required skill and if the employee does not work that particular assignment.

$$
\begin{array}{ll}
z_{i m d j} \leq 1-\sum_{m^{\prime} \in G} \sum_{s \in T_{d j}^{\prime \prime}} x_{i m^{\prime}(d+1) s}^{w} & \forall i \in N, \forall m \in G, \forall d \in D, \forall j \in S \\
z_{i m d j} \leq 1-\sum_{m^{\prime} \in G} \sum_{s \in T_{d j}^{\prime}} x_{i m^{\prime}(d-1) s}^{w} & \forall i \in N, \forall m \in G, \forall d \in D, \forall j \in S
\end{array}
$$


Equation (9) ensures that there is no substitution possibility with another shift that would violate the minimum rest period with the assignment on the next day (Eq. 9a) or the assignment on the previous day (Eq. 9b).

$$
\sum_{m \in G} \sum_{j \in S} z_{i m d j} \leq \epsilon \quad \forall i \in N, \forall d \in D
$$

Equation (10) limits the daily number of substitution possibilities each employee has to offer to $\epsilon$ and helps to model the way the substitution possibilities are counted.

$$
\sum_{i \in N} z_{i m d j} \leq \beta_{m d j} \quad \forall m \in G, \forall d \in D, \forall j \in S
$$

Equation (11) is a constraint that limits the number of substitution possibilities with respect to a particular shift, day and skill. This constraint helps to model how substitution possibilities should be included in the baseline personnel shift roster.

$$
\begin{array}{ll}
x_{i m d j}^{w} \in\{0,1\} & \forall i \in N, \forall m \in G, \forall d \in D, \forall j \in S \\
x_{i d}^{v} \in\{0,1\} & \forall i \in N, \forall d \in D \\
x_{m d j}^{w u} \geq 0 & \forall m \in G, \forall d \in D, \forall j \in S \\
z_{i m d j} \in\{0,1\} & \forall i \in N, \forall m \in G, \forall d \in D, \forall j \in S
\end{array}
$$

Constraints (12) embody the integrality conditions.

The algorithm to optimise this problem is discussed in Sect. 4. More information on how this model is employed to increase the employee substitutability and to obtain a good proxy of the operational decision phase in the tactical scheduling phase is provided in Sect. 5.1.

\subsubsection{Group employee substitutability}

We investigate group employee substitutability by requiring an additional number of employees to be on duty that are able to carry out a specific skill on top of the minimum staffing requirements during a particular shift. In line with the research of Campbell (1999) and Olivella and Nembhard (2016), employee substitutability on a group level is highly impacted by the degree of cross-training of the working employees as a higher number of skilled workers on duty can be obtained in different ways. First, more skilled employees are assigned to the specific duty than minimum required and a capacity buffer is created. The appropriate buffer size and positioning of capacity buffers has been investigated by Ingels and Maenhout (2015). Second, a higher number of skilled employees is attained by assigning multi-skilled employees to other skillduties during the same shift. The selected option is dependent upon the imposed constraints and the trade-off in the objective function, i.e. the extra wage cost for scheduling an additional duty on top of the minimum staffing requirements versus the cost of scheduling a more expensive multi-skilled worker. As such, the provided group 
employee substitutability gives an indication of the available between-skill, withinskill and day-off-to-work substitutions in the operational allocation phase. When the additional required number of skilled employees (i.e. the group staffing requirements) is low, a smaller number of workers are on duty and/or the more expensive multi-skilled employees are preferably assigned to a day off. As such, the baseline personnel shift roster embeds a higher flexibility in terms of day-off-to-work substitutions. When the additional required number of skilled employees increases, a higher number of multiskilled workers are assigned and a higher flexibility is introduced in terms of betweenand within-skill substitutions.

We formulate group employee substitutability in the following mathematical model, i.e.

$$
\begin{aligned}
\min & \sum_{i \in N} \sum_{m \in G} \sum_{d \in D} \sum_{j \in S}\left(c_{i m d j}^{w}+p_{i d j}\right) x_{i m d j}^{w}+\sum_{m \in G} \sum_{d \in D} \sum_{j \in S} c_{m d j}^{w u} x_{m d j}^{w u} \\
& +\sum_{m \in G} \sum_{d \in D} \sum_{j \in S} c_{m d j}^{w u,+} x_{m d j}^{w u,+}
\end{aligned}
$$

Similar to the problem that considers individual employee substitutability (Sect. 3.1.1), we optimise the personnel assignment costs and the understaffing of the staffing requirements (Eq. 13a). Additionally, we minimise the deviation from the desired degree of group employee substitutability, i.e. the shortage in the desired number of employees on duty able to carry out a specific skill (Eq. 13b).

$$
\sum_{i \in N} b_{i m} \sum_{m^{\prime} \in G} x_{i m^{\prime} d j}^{w}+x_{m d j}^{w u,+} \geq R_{m d j}^{w}+R_{m d j}^{w,+} \quad \forall m \in G, \forall d \in D, \forall j \in S
$$

Equation (14) determines the required number of employees on duty with a skill $m$ during shift $j$ on day $d$, which is the sum of the minimum staffing requirements and the additional group staffing requirements. In contrast to the minimum staffing requirements (Eq. 2), where each employee is accounted to carry out a single duty and skill along with his assignment, the group staffing requirements (Eq. 14) consider the overall number of skilled employees on duty during a particular shift since employees with multiple skills contribute to multiple skill categories. As such, this equation specifies the employee substitutability on a group level and the available degree of cross-training of the assigned workforce.

Note that this equation, which aims to improve the flexibility of the personnel shift roster in a multi-skilled operating environment via an increase of the staffing requirements for a specific skill, is conceptually based on the work of Ingels and Maenhout (2015). These authors define extra staffing requirements to include reserve duties in a single-skilled operating environment such that the stability of the resulting personnel shift roster is improved.

Apart from the cross-training objective and related constraints, we also include the minimum staffing requirements (Eq. 2) and the time-related constraints imposed on an employee schedule (Eqs. 3-7) in this model. 


$$
\begin{aligned}
& x_{i m d j}^{w} \in\{0,1\} \quad \forall i \in N, \forall m \in G, \forall d \in D, \forall j \in S \\
& x_{i d}^{v} \in\{0,1\} \quad \forall i \in N, \forall d \in D \\
& x_{m d j}^{w u} \geq 0 \quad \forall m \in G, \forall d \in D, \forall j \in S \\
& x_{m d j}^{w u,+} \geq 0 \quad \forall m \in G, \forall d \in D, \forall j \in S
\end{aligned}
$$

Constraints (15) embody the integrality conditions.

\subsection{Operational allocation phase}

In this short-term phase, we consider the baseline personnel shift roster on a day-byday basis. In order to imitate this phase, we first simulate the ad hoc variability for one particular day, which potentially leads to unexpected changes in the minimum staffing requirements and employee availability (Sect. 3.2.1). Second, the simulated variability may require adjustments to the baseline personnel shift roster to restore its workability and/or feasibility (Sect. 3.2.2). We repeat these two steps for each day in the baseline personnel shift roster. Moreover, this process of daily simulations and adjustments is executed several times such that we actually investigate multiple scenarios that may occur in reality and have an accurate idea of the expected performance for a particular baseline personnel shift roster. In the computational experiments described in Sect. 5, this process is executed 100 times.

\subsubsection{Simulation of operational variability}

We imitate the ad hoc variability through a discrete-event simulation of the uncertainty of demand $\left(R_{m d j}^{\prime} w\right)$ and the uncertainty of capacity $\left(a_{i d}\right)$ for the day under consideration, i.e. the demand and absenteeism are known at the start of day $d$.

Uncertainty of demand: We assume that the demand is Poisson distributed (Ahmed and Alkhamis 2009; Ingels and Maenhout 2015; Yeh and Lin 2007) and simulate the minimum staffing requirements for each shift and skill category on day $d$. The expected staffing requirement $\left(R_{m d j}^{w}\right)$ corresponds to the parameter $\lambda$ of the Poisson distribution.

Uncertainty of capacity: The uncertainty of capacity is simulated using a Bernoulli distribution for each employee. The probability of absenteeism $\left(P_{i d}(X=0)\right)$ determines whether the employee is available $\left(a_{i d}=1\right)$ or not $\left(a_{i d}=0\right)$. This probability $\left(P_{i d}(X=0)\right)$ depends on the basic probability of absenteeism $(P(X=0))$ and the number of days the employee has already been unexpectedly absent before day $d$ (Ingels and Maenhout 2015). The actual probability $\left(P_{i d}(X=0)\right)$ for an employee therefore decreases as the number of absent days increases for this employee. The basic probability of absence $(P(X=0))$ is $2.44 \%$ for organisations in Belgium (SD Worx 2013), which is consistent with the findings for Europe (European Foundation for the Improvement of Living and Working Conditions 2010) and the USA (Bureau of Labor Statistics 2013). 


\subsubsection{Operational adjustments}

When schedule disruptions occur as a result of the operational variability, the personnel planner needs to efficiently and effectively adjust the assignments of the employees on day $d$. This means that the disruption should be resolved by executing a number of adjustments in the cheapest way with a minimal impact on the baseline personnel shift roster. The mathematical formulation of this operational decision model is added in 'Appendix'. The recourse structure to deal with schedule disruptions contains the following reactive strategies:

- The reassignment of duties scheduled in the baseline personnel shift roster is the common reactive allocation strategy that is applied for schedule recovery. We consider the following types of reassignments: (a) a between-skill substitution, (b) a within-skill substitution and (c) a day-off-to-work substitution.

- The cancellation of duties that are superfluous on top of the actual demand for staff.

Adjustments to the baseline personnel shift roster are allowed as long as all time-related constraints remain satisfied (Eqs. 4-7). The available adjustments therefore depend on the assignments before and after day $d$. The assignments before day $d$ correspond to the assignments that were previously made in the operational allocation phase. The assignments after day $d$ correspond to the original assignments in the baseline personnel shift roster. Thus, we update the personnel shift roster on day $d$ after the execution of the operational adjustments and repeat the operational allocation phase for day $d+1$.

\subsection{Robustness evaluation}

We evaluate the robustness of the personnel shift rosters based on the planned and expected actual performance. The planned performance represents the quality of the baseline personnel shift roster in the tactical scheduling phase. The expected actual performance evaluates the quality of the personnel shift roster after the operational allocation phase. We make a distinction between the worst-case, average and bestcase performance over the different simulated operational scenarios (cf. Sect. 3.2). In this respect, we do not define robustness solely based upon the worst-case scenario as defined by Soyster (1973). A personnel shift roster constructed for the worst-case scenario is not representative for more realistic operational scenarios. This is due to the fact that too much cost is incurred for extra robustness in the tactical scheduling phase (Bertsimas and Sim 2004). It is therefore important to find a good balance between the level of conservatism in terms of robustness and the corresponding cost (Bertsimas and Thiele 2006), which explains why we make use of an expected value criterion to evaluate a baseline personnel shift roster.

Table 1 displays the different components and subcomponents based upon which the planned and expected actual performance is assessed.

The planned performance is defined by the planned cost, the planned substitutability value or the planned cross-training, and the planned overstaffing corresponding to the personnel shift roster constructed in the tactical scheduling phase (Sect. 3.1). The 
Table 1 Building blocks of the planned and expected actual performance

\begin{tabular}{ll}
\hline Planned performance & Expected actual performance \\
\hline Planned cost & Expected actual cost \\
Shortages (in shifts) & Shortages (in shifts) \\
Total assignment cost & Total assignment cost \\
Wage cost & Wage cost \\
Preference penalty cost & Preference penalty cost \\
& Number of duties cancelled \\
\hline Planned substitutability value & Expected utilised substitutions \\
Between-skill substitutions & Between-skill substitutions \\
Within-skill substitutions & Within-skill substitutions \\
Day-off-to-work substitutions & Day-off-to-work substitutions \\
\hline
\end{tabular}

Planned cross-training

Available cross-training $(C T)$

Cross-training surplus $\left(C T^{+}\right)$

Cross-training shortage $\left(C T^{-}\right)$

Planned overstaffing

Expected actual overstaffing

planned cost is related to the number of shortages and the total assignment cost, i.e. the wage cost and the preference penalty cost. The planned substitutability value is determined by the value and number of between-skill, within-skill and day-off-to-work substitution possibilities. We define the available cross-training, cross-training surplus and cross-training shortage in Eqs. (16), (17) and (18), respectively. The cross-training surplus (shortage) indicates the surplus (shortage) of skills of the working employees compared to the minimum staffing requirements.

$$
\begin{aligned}
C T & =\sum_{i \in N} \sum_{m \in G} b_{i m} \sum_{d \in D}\left(1-x_{i d}^{v}\right) \\
C T^{+} & =\sum_{m \in G} \sum_{d \in D} \sum_{j \in S} \max \left(\sum_{i \in N} b_{i m} \sum_{m^{\prime} \in G} x_{i m^{\prime} d j}^{w}-R_{m d j}^{w}, 0\right) \\
C T^{-} & =\sum_{m \in G} \sum_{d \in D} \sum_{j \in S} \max \left(R_{m d j}^{w}-\sum_{i \in N} b_{i m} \sum_{m^{\prime} \in G} x_{i m^{\prime} d j}^{w}, 0\right)
\end{aligned}
$$

The expected actual performance measures the quality of the personnel shift roster after the execution of the operational allocation phase for each day in the roster. The building blocks comprising the expected actual performance are similar to those of the planned performance but there are important differences. First, the cancelled duties are incorporated in the expected actual cost. Second, instead of listing the number of substitution possibilities for the planned performance, we list the number of adjustments or the utilised substitution possibilities in the operational phase. Since adjustments are made on the level of an individual employee in the operational phase, 
these metrics are also used to evaluate the personnel shift rosters with optimised substitutability on the group level.

\section{A two-phase pre-emptive programming approach for individual employee substitutability}

In the tactical scheduling phase, we propose a pre-emptive programming approach to construct medium-term personnel shift rosters. In this respect, we utilise a two-phase methodology to solve model (1)-(12) as follows, i.e.

- In the first phase, we focus solely on cost minimisation. As such, we construct the minimum cost personnel shift roster by solving the model comprising the cost objective (Eq. 1a), the minimum staffing requirements (Eq. 2) and the timerelated constraints (Eqs. 3-7). Equation (1a) determines the total cost ( $\left.c^{\text {total }}\right)$ of the constructed roster.

- In the second phase, we adapt the minimum cost personnel shift roster and focus on the maximisation of the value of individual employee substitutability. We solve a model that consists of the substitutability objective (Eq. 1b), minimum staffing requirements (Eq. 2), time-related constraints (Eqs. 3-7) and substitutability constraints (Eqs. 8-11). Moreover, we add a constraint to limit the total cost (Eq. 19), i.e. the cost of the robust personnel shift roster may not exceed $c^{\text {total }} \times(1+\tau)$. This cost constraint enables the management of the allowable cost increase $(\tau)$ and facilitates the investigation of the trade-off between the additional flexibility and the cost of this extra robustness, i.e. the cost difference between the minimum cost personnel shift roster (first phase) and the more robust personnel shift roster (second phase) (Bertsimas and Sim 2004).

$$
\sum_{i \in N} \sum_{m \in G} \sum_{d \in D} \sum_{j \in S}\left(c_{i m d j}^{w}+p_{i d j}\right) x_{i m d j}^{w}+\sum_{m \in G} \sum_{d \in D} \sum_{j \in S} c_{m d j}^{w u} x_{m d j}^{w u} \leq c^{\text {total }}(1+\tau)
$$

These two phases are solved separately and result in a personnel shift roster with a maximal employee substitutability value for a given cost of robustness. The first phase is solved to optimality by applying a branch-and-bound procedure with the commercial optimisation software (Gurobi 2014). For the second phase we employ a dedicated procedure to maximise employee substitutability since commercial software fails to provide optimal solutions within a reasonable timeframe (3600s). The applied procedure consists of a truncated branch-and-bound procedure followed by a branchand-price procedure, i.e.

- The truncated branch-and-bound procedure aims to improve the minimum cost solution by constructing a better incumbent solution. In this respect, a branch-andbound thriving on commercial software (Gurobi 2014) is truncated after reaching a time limit of 120s. This results in a strong upper bound and is input to the branch-and-price procedure to speed up its performance.

- Next, we apply a branch-and-price procedure (Ingels and Maenhout 2015; Maenhout and Vanhoucke 2010) to improve the MIP-gap between the lower and upper bound. This procedure is stopped when the MIP-gap shrinks to a value below $0.1 \%$. 
Since this gap is very small, we are able to report optimal and/or near-optimal solutions in a reasonable timeframe.

\section{Computational experiments}

In this section, we provide computational insight into our methodology to improve the robustness of personnel shift rosters. In Sect. 5.1, we describe the characteristics of the test problem instances and discuss the parameter settings of the optimisation models. We evaluate the planned and average expected actual performance of different strategies to model employee substitutability in Sect. 5.2. In this section, we consider a full-factorial test design that consists of a set of artificially generated test instances. As such, we analyse the impact of different demand profiles and skill possession settings of the workforce to provide specific guidelines to increase the robustness of a personnel shift roster. In Sect. 5.3, we investigate the variability of the obtained results by considering the expected best-case, average and worst-case actual performance to validate the robustness provided by three scheduling strategies for constructing a baseline personnel shift roster, i.e. the minimum cost, individual employee substitutability and group employee substitutability strategy. In order to confirm the delivered robustness in a real-life setting, we focus on test instances with a real-life demand profile. All tests were carried out on an Intel Core processor 2.5 GHz and 4GB RAM. Model (1)-(12) is solved by the proposed pre-emptive programming approach to construct a personnel shift roster with an optimised individual employee substitutability. The average CPU time is 354 seconds with a median of 3.13 seconds. This means that we observe a number of outliers that require a high CPU time to obtain an average optimality gap of $0.0015 \%$. Other models were solved to optimality within smaller CPU times.

\subsection{Test design}

In this section, we provide detailed information on the full-factorial design of generated problem instances (Artificial set) and the characteristics of a set of instances based on a real-life demand profile (Real-life set). In addition, we discuss the underlying parameter settings of the personnel and shift characteristics (Sect. 5.1.1), the constraints (Sect. 5.1.2) and the objectives (Sect. 5.1.3) of the different models.

\subsubsection{Personnel and shift characteristics}

The artificial test instances consist of 20 employees and have a planning horizon of 28 days, while the real-life test instances consist of a varying number of employees and a 30-day planning horizon.

Personnel characteristics-Both sets of instances contain a maximum of 2 skills for each employee. In total, we categorise 11 skill possession settings according to the triplet $\left(m_{1} \%-m_{2} \%-m_{1,2} \%\right)$. This triplet indicates the percentage of employees who uniquely possess skill $1\left(m_{1} \%\right)$, skill $2\left(m_{2} \%\right)$ or both skills $\left(m_{1,2} \%\right)$. We distinguish skill possession settings varying between $(50 \%, 50 \%, 0 \%)$ and $(0 \%, 0 \%, 100 \%)$ with intervals of $5 \%$ for $m_{1}$ and $m_{2}$ and an interval of $10 \%$ for $m_{1,2}$. In this respect, $m_{1,2} \%$ represents the degree of cross-training of the workforce. 
Shift characteristics-The artificial and real-life problem instances are characterised by three non-overlapping shifts with specific start and end times. These shifts have a fixed duration of 8 hours and, respectively, start at 6 a.m., 2 p.m. and 10 p.m.

\subsubsection{Constraint parameter settings}

Time-related constraints-All personnel members can perform only a single working assignment per day (Eq. 3). There is also a minimum rest period of 11 hours imposed between two working assignments (Eq. 4). Furthermore, we include the following time-related constraints with their corresponding parameter values:

- The maximum number of working hours $\left(\eta_{i}^{w, \max }\right)$ is 160 and 168 (Eq. 5) for the artificial and real-life sets, respectively.

- The minimum number of working hours $\left(\eta_{i}^{w, \min }\right)$ is 128 and 136 (Eq. 6) for the artificial and real-life sets, respectively.

- The maximum number of consecutive working assignments per employee $\left(\theta_{i}^{w, \max }\right)$ is 5 (Eq. 7) for the artificial and real-life sets.

Note that, in this research study, we want to obtain an unbiased insight into the impact of substitutability on the robustness of a personnel shift roster. To that purpose, we deliberately limit the number of time-related constraints in both optimisation models (1)-(12) and (13)-(15) to have some extra scheduling flexibility and a better match with the minimum staffing requirements.

Minimum staffing requirements of the artificial set-We consider a full-factorial design to define the profile of the staffing requirements for the artificial set of test instances. To obtain different demand profiles, we use the complexity indicators designed for the nurse shift scheduling problem (Vanhoucke and Maenhout 2009). In their paper, Vanhoucke and Maenhout (2009) define three complexity indicators whose values range between 0 and 1, i.e.

- The Total Coverage Constrainedness (TCC) expresses the ratio between the staffing requirements aggregated over the planning horizon and the theoretical maximum number of possible working assignments (=the number of employees $x$ the number of days). We generate instances with a TCC value of $0.30,0.40$ and 0.50 and distribute these staffing requirements evenly over the two skill categories.

- The Day Coverage Distribution (DCD) reflects the variability of the staffing requirements over the days of the planning horizon. A value of 1 indicates maximum variability over the days, while a value of 0 evenly distributes the requirements over the days. We investigate test instances with a value of 0.00 , 0.25 and 0.50 .

- The Shift Coverage Distribution (SCD) reflects the variability of the staffing requirements over the shifts for a single day. Values of 1 and 0 , respectively, indicate maximum variability and an even distribution of the staffing requirements over the shifts of a single day. The staffing requirements are generated with SCD values of $0.00,0.25$ and 0.50 .

Each combination of values for these complexity indicators leads to a specific demand profile. We generate instances for $27(3 \times 3 \times 3)($ TCC, DCD, SCD $)$-combinations 
and 11 skill possession settings, which implies that we discuss the most significant results over $297(=27 \times 11)$ artificial instances.

Minimum staffing requirements of the real-life set-We highlight the practical relevance of employee substitutability by extending our test set with a number of instances that consider real-life demand profiles. These profiles are based on the data set of Ikegami and Niwa (2003), which is also available as a personnel rostering benchmark problem ${ }^{1}$ (Brucker et al. 2010). The authors investigate the nurse shift scheduling problem in Japan and provide lower and upper bounds on the staffing requirements for two teams of nurses with different skills. These lower and upper bounds provide real-life demand profiles for which we investigate different staffing levels. These staffing levels vary between 27 and 67 workers and are based on a TCC value ranging between 0.30 and 0.50 . These demand profiles are investigated in conjunction with 11 different skill possession settings, which results in a total of 187 realistic test instances.

Additional group staffing requirements-The parameter $R_{m d j}^{w,+}$ defines the number of skilled employees that are required to be on duty on top of the minimum staffing requirements. This parameter gives an indication of the group employee substitutability as explained in Sect. 3.1.2. In correspondence with Ingels and Maenhout (2015), we define $R_{m d j}^{w,+}$ as a fixed ratio of the minimum staffing requirements $\left(R_{m d j}^{w}\right)$ and distinguish a ratio of $0,25,50,75$ and $100 \%$.

Individual employee substitutability constraints-Constraints (8)-(11) define the employee substitutability in the tactical decision phase. These constraints impose restrictions on the counted number of substitution possibilities in order to obtain a better estimate of the real substitutability in the operational decision phase. The righthand side parameters $\epsilon$ and $\beta_{m d j}$ allow the definition of different strategies to count the number of substitution possibilities and to position them appropriately. By experimenting with these strategies, we aim to include the best proxy of the operational allocation phase in the tactical decision process to construct a baseline personnel shift roster that embeds a higher flexibility.

Constraint (10) reflects the number of substitution possibilities an employee may offer on a single day and $\epsilon$ may be defined as follows:

$-\epsilon=1$ : As a single employee on a particular day may only be reassigned to one other duty in the operational allocation phase, at most one substitution possibility is counted in the tactical scheduling phase.

$-\epsilon=|G| \times|S|$ : A single duty or day-off assignment for an employee may offer multiple substitution possibilities to each other feasible (skill, shift)-combination. The maximum number of substitution possibilities is equal to the number of (skill, shift)-combinations.

Constraint (11) reflects the number of substitution possibilities that may be counted per duty, i.e. for a single (skill, day, shift)-combination, and $\beta_{m d j}$ may be defined as follows:

$-\beta_{m d j}=R_{m d j}^{w}$ : The number of substitution possibilities is limited to the minimum staffing requirements of the particular duty. This implies that the scheduling of

\footnotetext{
${ }^{1}$ http://www.cs.nott.ac.uk/ psztc/NRP/.
} 
Table 2 An overview of the substitution counting strategies

\begin{tabular}{lll}
\hline Counting strategy & $\epsilon$ & $\beta_{m d j}$ \\
\hline Strategy 1.1 & 1 & $R_{m d j}^{w}$ \\
Strategy 1.2 & 1 & $\sum_{i \in N} x_{i m d j}^{w}$ \\
Strategy 1.3 & 1 & $M$ \\
\hline Strategy 2.1 & $|G| \times|S|$ & $R_{m d j}^{w}$ \\
Strategy 2.2 & $|G| \times|S|$ & $\sum_{i \in N} x_{i m d j}^{w}$ \\
Strategy 2.3 & $|G| \times|S|$ & $M$ \\
\hline
\end{tabular}

additional and/or excess employees will have no impact on the number of substitution possibilities.

- $\beta_{m d j}=\sum_{i \in N} x_{i m d j}^{w}$ : The number of substitution possibilities is limited to the number of assigned employees. Hence, each scheduled employee can be substituted by a maximum of one other employee.

- $\beta_{m d j}=M$ : No limitation is imposed on the number of substitution possibilities (with $M=$ a very large number). Each assigned employee can be substituted by an unlimited number of other employees.

Based upon the parameter values of $\epsilon$ and $\beta_{m d j}$, different strategies can be defined to count the number of substitution possibilities and to mimic the substitutability in the operational phase. The different counting strategies refer to the set of potential substitution possibilities each assignment for a specific employee may offer. These strategies are displayed in Table 2 and are assessed in Sect. 5.2 for different values of the allowable cost increase $(\tau)$ to obtain insight into the cost of robustness.

\subsubsection{Objective function parameter settings}

General objective function-The general objective in models (1)-(12) and (13)-(15) is to optimise the costs, i.e. the personnel assignment costs and the understaffing of the minimum staffing requirements (Eqs. 1a and 13a). For these general objective function components, we define the following parameter values, i.e.

- Every employee has a wage cost $\left(c_{i m d j}^{w}\right)$ depending on the number of skills this employee possesses (Bruecker et al. 2015), i.e. $10 \times 1.2^{\sum_{m \in G} b_{i m}-1}$.

- Every employee has a preference penalty cost $\left(p_{i d j}\right)$ that is randomly generated in the range of 1 to 5 .

- The shortage cost $\left(c_{m d j}^{w u}\right)$ is fixed at 20 .

The objective to optimise the individual employee substitutability-In order to optimise the employee substitutability on the level of the individual employee, we define a value for the objective function coefficient $\gamma_{m d j}$ (Eq. 1b) based on three different strategies, i.e. 
- Strategy 1: Each substitution embodies a fixed value $(\gamma)$ independent of the skill category, day and shift (Eq. 20).

$$
\gamma_{m d j}^{1}=\gamma \quad \forall m \in G, \forall d \in D, \forall j \in S
$$

- Strategy 2: The weight for a substitution possibility associated with a shift and skill category depends on how the staffing requirements are related to the maximum staffing requirements over all shifts for the skill category on that day (Eq. 21).

$$
\gamma_{m d j}^{2}=\frac{R_{m d j}^{w}}{\max _{s \in S} R_{m d s}^{w}} \times \gamma \quad \forall m \in G, \forall d \in D, \forall j \in S
$$

- Strategy 3: This strategy calculates the ratio of the staffing requirements for a shift, day and skill category to the maximum staffing requirements over the total planning horizon for the skill category (Eq. 22).

$$
\gamma_{m d j}^{3}=\frac{R_{m d j}^{w}}{\max _{d^{\prime} \in D, s \in S} R_{m d^{\prime} s}^{w}} \times \gamma \quad \forall m \in G, \forall d \in D, \forall j \in S
$$

These value strategies try to value the substitution possibilities in such a way that the most valuable, in terms of the expected performance in the operational allocation phase, are added during the construction of the personnel shift roster in the tactical scheduling phase.

The objective to optimise the group employee substitutability-In order to optimise the employee substitutability on the group level, we define a value for the objective function coefficient $c_{m d j}^{w u,+}$ (Eq. 13b) that amounts to 25. This value exceeds the cost of a shortage of employees for a skill, day and shift $\left(c_{m d j}^{w u}\right)$ to express that it is worse to have a shortage of skills on a group level rather than on the level of an individual employee. Hence, this value accommodates the satisfaction of the additional group staffing requirements defined in Eq. (14).

\subsection{Comparison of strategies to model employee substitutability}

In this section, we discuss and compare the planned and average expected actual performance of different proactive scheduling strategies for the artificial set of instances. In Sect. 5.2.1, we consider employee substitutability on the level of the individual employee and analyse the impact of the cost of robustness for different counting strategies, the value strategies and the substitution types. In Sect. 5.2.2, we consider employee substitutability on a group level and assess the benefits of different sizes of the group staffing requirements.

\subsubsection{Employee substitutability on an individual employee level}

The constructed personnel shift rosters differ based on the allowable cost increase $(\tau)$ and the applied counting strategies, the value strategies and the impact of the 
Table 3 The planned cost for different counting strategies and allowable cost increases $(\tau)$

\begin{tabular}{lllll}
\hline Counting strategy & $\tau=0 \%$ & $\tau=1 \%$ & $\tau=2 \%$ & $\tau=3 \%$ \\
\hline Strategy 1.1 & 3981.29 & 3981.29 & 3981.29 & 3981.29 \\
Strategy 1.2 & 3981.29 & 3988.62 & 3988.69 & 3988.69 \\
Strategy 2.3 & 3981.29 & 4020.57 & 4058.88 & 4082.35 \\
\hline
\end{tabular}

individual substitution types. Unless otherwise stated, we discuss the average results over all demand profiles and skill possession settings.

Impact of the counting strategy and the cost of robustness

Table 3 indicates the planned cost of the three most relevant counting strategies given different values for the allowable cost increase $(\tau)$. These counting strategies include the most and least restrictive method to count substitution possibilities, i.e. strategy 1.1 and 2.3, respectively. Moreover, we add the counting strategy with the best expected actual performance in the operational allocation phase, i.e. strategy 1.2. Note that all other counting strategies exhibit a performance that can be situated between strategy 1.1 and 2.3 .

It is clear that the impact of the allowable cost increase $(\tau)$ on the planned cost strongly depends on the way the substitution possibilities are counted. In this respect, the planned cost associated with strategy 2.3 rises with the allowed cost increase, while strategy 1.1 results in a stable planned cost. This difference is due to the definition of the corresponding values for $\epsilon$ and $\beta_{m d j}$ in constraints (10) and (11) (cf. Table 2). Counting strategy 2.3 does not impose a limit on the counted number of substitution possibilities for each employee and duty in the constructed baseline personnel shift roster. This number can only be increased by changing the position of surplus duties between shifts or by creating more planned overstaffing or capacity buffers. This results in an increase of the preference penalty cost and/or wage cost and in a larger cost of robustness, i.e. the cost difference between the minimum cost and the more robust personnel shift roster. Counting strategy 1.1 in contrast, has a stringent upper limit on the counted number of substitution possibilities and is therefore not affected by an increase of the allowable cost. As such, the maximum number of substitution possibilities that can be introduced is significantly smaller than for strategy 2.3 and the planned cost remains equal to the cost of the minimum cost personnel shift roster, i.e. $\tau=0 \%$. Given the extra flexibility offered by strategy 1.2 , the cost of robustness rises due to a small increase in the total assignment cost. This is the result of a change in the assignments to increase the number of substitution possibilities.

The allowable cost increase and the counting strategy have a substantial impact on the expected performance of the personnel shift roster in the operational allocation phase. We display the average expected actual cost of the three counting strategies in Table 4. Given that the average expected actual cost of the minimum cost personnel shift roster equals 4193.61 , this table shows that the counting strategies are able to provide an improvement when the cost of robustness is 0 , i.e. $\tau=0 \%$.

The significance of this improvement depends on the selected counting strategy. Irrespective of the allowable cost increase $(\tau)$, it is clear that the average expected 
Table 4 The average expected actual cost for different counting strategies and allowable cost increases $(\tau)$

\begin{tabular}{lllll}
\hline Counting strategy & $\tau=0 \%$ & $\tau=1 \%$ & $\tau=2 \%$ & $\tau=3 \%$ \\
\hline Strategy 1.1 & 4192.06 & 4192.09 & 4192.09 & 4192.09 \\
Strategy 1.2 & 4179.56 & 4177.11 & 4177.00 & 4176.98 \\
Strategy 2.3 & 4191.38 & 4220.82 & 4243.48 & 4255.76 \\
\hline
\end{tabular}

actual cost and the associated shortages, changes and cancellations are lower when the maximum number of substitution possibilities per duty $\left(\beta_{m d j}\right)$ are restricted to the number of scheduled employees (strategy 1.2). The expected actual cost actually decreases when the allowable cost increase $(\tau)$ rises but does not significantly change once an allowable cost increase of $1 \%$ has been reached. It is therefore most important to allow a slight increase in the planned costs to obtain more substitution possibilities with a different position. A more stringent restriction on the maximum number of substitution possibilities per duty (strategy 1.1) provides the smallest expected actual cost when the staffing requirements exhibit a small variability over the different days (i.e. a small DCD value), which is the case for the real-life staffing requirements. In this respect, counting strategy 1.1 ensures that a lower number of substitution possibilities can be better distributed over the days and shifts to enhance their position and to achieve a smaller number of shortages and cancellations in the operational allocation phase. As such, counting strategy 2.3 always leads to a significantly higher expected actual cost due to a poor positioning of an excessive number of substitution possibilities leading to a high number of shortages, changes and cancellations, which is especially true for a rising allowable cost increase $(\tau)$. The number of substitution possibilities that may be counted for one employee per day $(\epsilon)$ should generally be restricted. However, this number actually depends on the degree of cross-training of the workforce and the potential number of substitution possibilities available in the constructed personnel shift roster, i.e. the implicit flexibility. For a high number of multi-skilled employees, it is important to limit the number of substitution possibilities that may be counted per employee, i.e. $\epsilon=1$. This limitation facilitates a focus on the definition of the most important substitution possibilities in terms of their position in the personnel shift roster. In contrast, a low number of multi-skilled employees represents less implicit flexibility and more substitution possibilities should be added.

Therefore, a sufficient number of substitution possibilities should always be available in the operational allocation phase. This can be ensured by applying the adequate counting strategy and allowable cost increase $(\tau)$. In this respect, a lower number of multi-skilled employees and a smaller number of implicit substitution possibilities require a less restrictive counting strategy (strategy 2.2) in combination with a higher allowable cost increase $(\tau)$. As more substitution possibilities are implicitly available, the focus should move to a counting strategy that better reflects the actual situation in the operational allocation phase, i.e. each scheduled employee can be substituted by maximum one other employee and a single employee may only be reassigned to one other duty in the operational allocation phase (strategy 1.2). 
Table 5 The planned performance for different value strategies

\begin{tabular}{llll}
\hline & Strategy 1 & Strategy 2 & Strategy 3 \\
\hline Planned cost & 3981.91 & 3995.19 & 3988.62 \\
Shortages & 0.02 & 0.02 & 0.02 \\
Total assignment cost & 3981.57 & 3994.86 & 3988.28 \\
$\quad$ Wage cost & 3520.00 & 3520.00 & 3520.00 \\
Preference penalty cost & 461.57 & 474.86 & 468.28 \\
\hline Planned substitutability value & 476.73 & 406.25 & 293.68 \\
Between-skill substitutions & 73.94 & 75.41 & 67.11 \\
Within-skill substitutions & 124.88 & 117.72 & 122.51 \\
Day-off-to-work substitutions & 119.00 & 114.06 & 111.04 \\
\hline Planned overstaffing & 96.02 & 96.02 & 96.02 \\
\hline
\end{tabular}

\section{The impact of the value strategies}

Table 5 shows the planned performance corresponding to the defined value strategies (cf. Eqs. 20-22) for counting strategy 1.2 and an allowable cost increase $\tau$ of $1 \%$. Value strategy 1 , which defines constant substitution benefit values, exhibits the smallest planned cost and the highest substitutability value and number of substitution possibilities. In contrast, value strategy 2 leads to the largest planned cost.

The results in Table 5 represent personnel shift rosters constructed with a benefit value $\gamma$ equal to 1.50 , which results in the best expected actual performance. Note that a smaller (larger) value for this parameter leads to a lower (higher) planned cost and planned substitutability value.

Figure 2 displays the expected actual cost for the different value strategies. The best results are clearly obtained by applying a value strategy that considers the demand profile in the definition of the substitution benefit values, i.e. strategies 2 and 3. This confirms that the position of the substitution possibilities is of major importance. In this respect, value strategy 3 leads to the best positions and is especially useful when the staffing requirements exhibit a large variability over the days in the planning horizon (i.e. a large DCD value). In contrast, strategy 2 provides the best results when the staffing requirements exhibit a small variability over the days in the planning horizon, which is the case for the real-life staffing requirements.

Moreover, the substitution benefit value $\gamma$ has a significant impact on the weighting and positioning of the substitution possibilities and, consequently, on the expected actual performance of a baseline personnel shift roster. In this respect, the substitution benefit value should be set highest for those demand profiles with a high variability in the staffing requirements (i.e. high DCD and SCD values) when value strategy 2 or 3 is utilised. These strategies consider the demand profile and focus on those shifts with the highest staffing requirements. In order to ensure that shifts with smaller staffing requirements are also protected with some additional flexibility, the substitution benefit value $\gamma$ should be sufficiently high. 


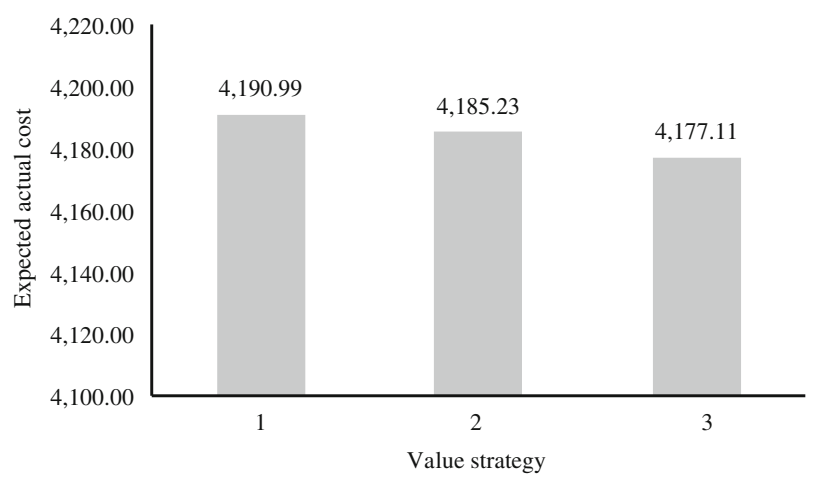

Fig. 2 The expected actual cost for different value strategies

\section{The impact of the individual substitution types}

The employee substitutability optimisation model (Eqs. (1)-(12)) concurrently considers the three types of substitutions. However, it is possible to adapt this model by adding constraint (23a), constraint (23b) or constraint (23c) to optimise employee substitutability solely based on between-skill substitutions, within-skill substitutions or day-off-to-work substitutions, respectively.

$$
\begin{aligned}
z_{i m d j} \leq \sum_{m^{\prime} \in G \backslash\{m\}} \sum_{j^{\prime} \in S} x_{i m^{\prime} d j^{\prime}}^{w} & \forall i \in N, \forall m \in G, \forall d \in D, \forall j \in S \\
z_{i m d j} \leq \sum_{j^{\prime} \in S \backslash\{j\}} x_{i m d j^{\prime}}^{w} & \forall i \in N, \forall m \in G, \forall d \in D, \forall j \in S \\
z_{i m d j} \leq x_{i d}^{v} & \forall i \in N, \forall m \in G, \forall d \in D, \forall j \in S
\end{aligned}
$$

Regardless of the considered substitution types, the general conclusions concerning the cost of robustness, counting strategies and value strategies remain valid. However, as a result of restricting the substitution types, the potential number of substitution possibilities available in the baseline personnel shift roster, i.e. the implicit flexibility, is smaller. In this respect, more substitution possibilities need to be explicitly added by applying counting strategy 2.2 in conjunction with a larger benefit value $\gamma$.

The individual substitution types only affect the planned performance of personnel shift rosters in terms of planned substitutability value and the associated number of substitution possibilities. However, they do have an important impact on the expected actual performance in the operational allocation phase. In this phase, a change cost $c_{i m d j}^{w \delta}$ is accounted if an assignment is adjusted, i.e. a substitution is executed. In Table 6 , we distinguish four operational change cost scenarios in relation to the considered substitution types in the tactical scheduling phase.

Figure 3 displays the expected actual cost for the individual and combined substitution types as a function of the degree of cross-training of the workforce. It is clear that the lowest expected actual cost and, hence, the highest flexibility are obtained when all substitution types are considered, which indicates that the substitution types are complementary. When we analyse the results in relation to the degree of cross- 
Table 6 The change cost $\left(c_{i m d j}^{w \delta}\right)$ for the different substitution types

\begin{tabular}{|c|c|c|c|}
\hline$c_{i m d j}^{w \delta}$ & $\begin{array}{l}\text { Between-skill sub- } \\
\text { stitution }\end{array}$ & $\begin{array}{l}\text { Within-skill sub- } \\
\text { stitution }\end{array}$ & $\begin{array}{l}\text { Day-off-to-work } \\
\text { substitution }\end{array}$ \\
\hline \multicolumn{4}{|c|}{ Considered substitutions } \\
\hline All & 1 & 1 & 1 \\
\hline Between-skill & 1 & 100 & 100 \\
\hline Within-skill & 100 & 1 & 100 \\
\hline Day-off-to-work & 100 & 100 & 1 \\
\hline
\end{tabular}

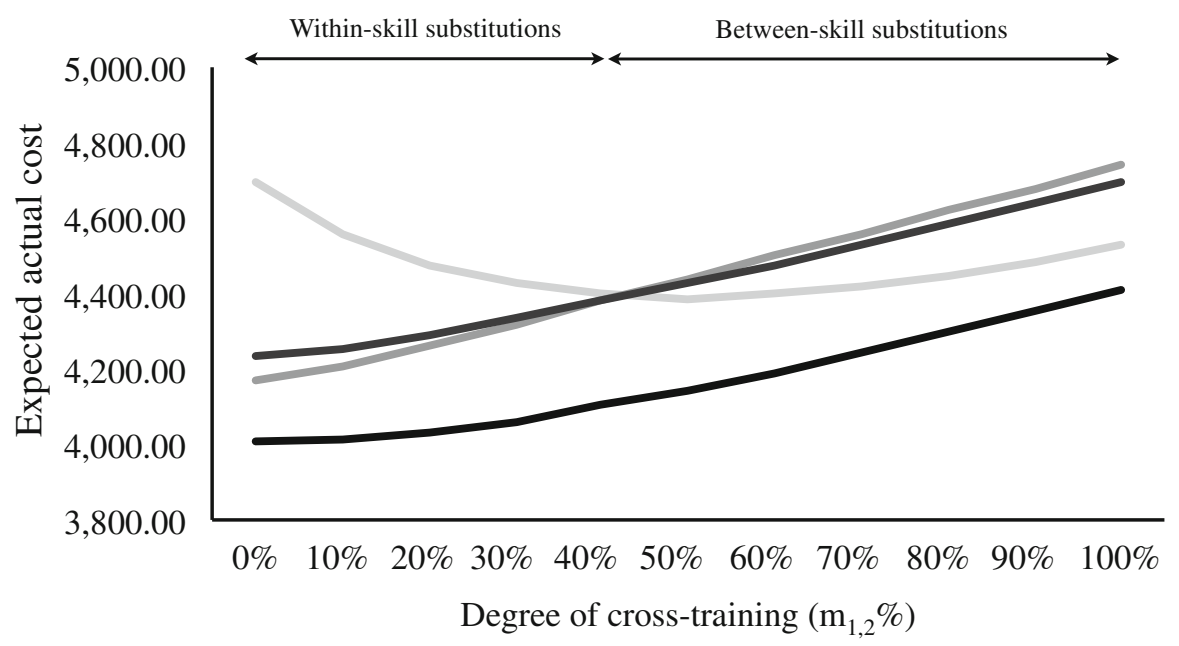

$\longrightarrow$ All $\longrightarrow$ Between-skill Within-skill $\longrightarrow$ Day-off-to-work

Fig. 3 The evolution of the expected actual cost for different substitution types and degrees of cross-training of the workforce

training, we observe that for a low number of multi-skilled employees, the within-skill substitutions offer the highest flexibility and have a better expected actual cost than the between-skill and day-off-to-work substitutions. As the number of multi-skilled employees rises, however, the between-skill substitutions offer the lowest expected actual cost and highest flexibility.

The figure clearly shows that the between-skill substitutions are unable to provide a good expected actual performance when the degree of cross-training is less than $50 \%$. This is due to the high number of staff shortages. As the degree of cross-training increases, the number of staff shortages decreases. However, since multi-skilled employees receive a higher wage than single-skilled employees, there is an increase in the expected actual cost when the degree of cross-training is more than $50 \%$. In contrast, when considering within-skill substitutions only, the expected actual cost steadily increases with a higher number of multi-skilled workers, whereas the num- 
ber of shortages remains rather constant. When focusing solely on day-off-to-work substitutions, the increase of the expected actual cost is smaller due to the decrease of the number of shortages when the degree of cross-training increases. The latter is the result of the larger flexibility embedded in the day-off assignments of employees, who can be reassigned to each duty, regardless of the corresponding skill. As such, the day-off-to-work substitutions outperform the within-skill substitutions for a large number of multi-skilled employees.

In general, we can conclude that it is important to exploit the multi-skilled nature of employees by considering between-skill and day-off-to-work substitutions. Additionally, within-skill substitutions provide extra flexibility in the personnel shift roster and are complementary to the other two substitution types.

\subsubsection{Employee substitutability on a group level}

Table 7 shows the planned performance associated with different sizes of the additional group staffing requirements $R_{m d j}^{w,+}$ calculated as a fixed ratio (i.e. $0 \%, 25 \%, 50 \%, 75 \%$ and $100 \%$ ) of the minimum staffing requirements $R_{m d j}^{w}$. In this respect, a ratio of $0 \%$ results in a personnel shift roster that represents the same planned cost as the minimum cost personnel shift roster (cf. Table 3). As the additional group staffing requirement rise, however, the planned cost increases due to a higher total assignment cost as a result of a higher number of working assignments and/or a higher number of assignments for multi-skilled workers. Note that the reported results in Table 7 represent the average over all skill possession settings and demand profiles (cf. Sect. 5.1). As such, it is not possible to avoid a certain level of understaffing (0.02) in some cases, which is caused by the hard minimum rest constraint (cf. Eq. 4).

Figure 4 shows that the overstaffing increases as the desired group staffing requirements increase. This is due to the fact that the group staffing requirements cannot be entirely satisfied by scheduling multi-skilled employees, especially if the number of multi-skilled employees is low. As such, additional working assignments are sched-

Table 7 The planned performance for different sizes of the additional group staffing requirements

\begin{tabular}{llllll}
\hline & $0 \%$ & $25 \%$ & $50 \%$ & $75 \%$ & $100 \%$ \\
\hline Planned cost & 3981.29 & 4037.27 & 4178.86 & 4363.30 & 4449.72 \\
Shortages & 0.02 & 0.02 & 0.03 & 0.02 & 0.04 \\
Total assignment cost & 3980.96 & 4036.86 & 4178.26 & 4362.83 & 4448.98 \\
$\quad$ Wage cost & 3520.00 & 3540.65 & 3631.49 & 3751.10 & 3808.90 \\
$\quad$ Preference penalty cost & 460.96 & 496.21 & 546.76 & 611.73 & 640.08 \\
\hline Planned cross-training & & & & & 510.46 \\
CT & 480.00 & 482.27 & 493.92 & 286.48 & 295.24 \\
CT ${ }^{+}$ & 256.02 & 258.29 & 269.94 & 0.02 & 0.03 \\
C $T^{-}$ & 0.02 & 0.02 & 0.02 & 117.30 & 122.35 \\
\hline Planned overstaffing & 96.02 & 98.03 & 106.49 & & 5.21 \\
\hline
\end{tabular}




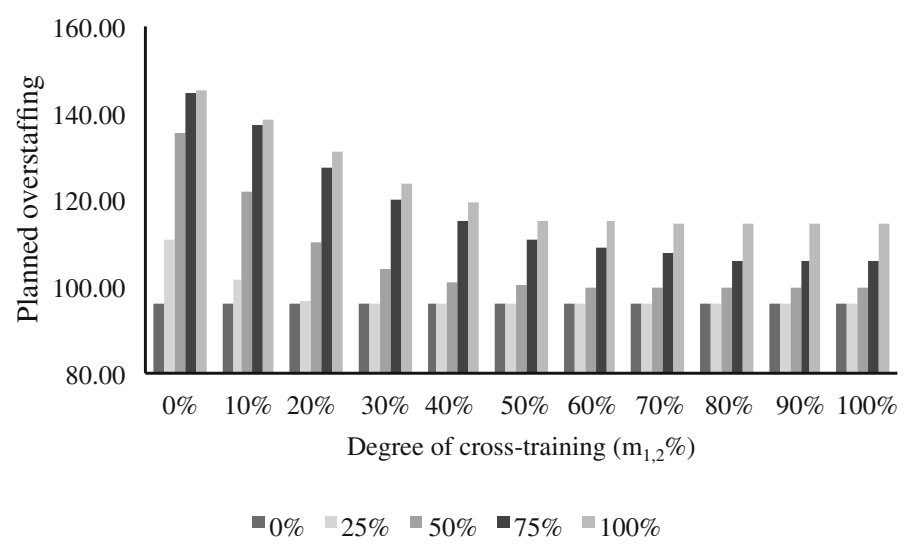

Fig. 4 The evolution of planned overstaffing for different sizes of the additional group staffing requirements and degrees of cross-training of the workforce

Table 8 The expected actual performance for different sizes of the additional group staffing requirements

\begin{tabular}{llllll}
\hline & $0 \%$ & $25 \%$ & $50 \%$ & $75 \%$ & $100 \%$ \\
\hline Expected actual cost & 4193.99 & 4188.43 & 4220.95 & 4277.24 & 4306.36 \\
Shortages & 3.91 & 3.75 & 3.84 & 4.07 & 4.16 \\
Total assignment cost & 4055.88 & 4057.50 & 4091.98 & 4145.65 & 4173.18 \\
Wage cost & 3455.75 & 3452.42 & 3446.72 & 3439.47 & 3436.98 \\
Preference penalty cost & 530.24 & 538.48 & 551.65 & 570.69 & 578.87 \\
Number of duties cancelled & 13.98 & 13.32 & 18.72 & 27.10 & 31.47 \\
\hline Expected utilised substitutions & 59.91 & 55.90 & 52.10 & 50.12 & 50.01 \\
Between-skill substitutions & 19.47 & 20.47 & 20.97 & 21.06 & 21.18 \\
Within-skill substitutions & 24.86 & 22.79 & 21.86 & 22.65 & 23.20 \\
Day-off-to-work substitutions & 15.59 & 12.64 & 9.27 & 6.40 & 5.64 \\
\hline Expected actual overstaffing & 93.58 & 93.09 & 92.65 & 92.22 & 92.08 \\
\hline
\end{tabular}

uled and create capacity buffers that augment the available cross-training $(C T)$ and cross-training surplus $\left(C T^{+}\right)$.

The average expected actual performance of personnel shift rosters for different sizes of the additional group staffing requirements is displayed in Table 8. In this table, we report the results obtained by considering all substitution types (cf. Table 6) and show that the best expected performance is obtained with an additional group staffing requirement ratio of $25 \%$, for which we observe the smallest number of shortages and cancellations. Larger and smaller ratios result in more shortages and cancellations. The cancellations are especially large for high group staffing requirements in combination with a small number of multi-skilled employees, which indicates a poor positioning of excessive capacity buffers. Moreover, the expected number of utilised substitutions decreases as the size of the additional group staffing requirements rises, which is mainly due to a reduction in the day-off-to-work substitutions. 
It is also important to note that the size of the additional group staffing requirements depends on the ratio between the total minimum staffing requirements $\left(R_{m d j}^{w}\right)$ and the total number of hired employees, i.e. the TCC value. As the total staffing requirements rise or the number of hired employees declines, the optimal size actually decreases because a large TCC value in combination with a high desired size actually results in substantial group staffing requirements $\left(R_{m d j}^{w,+}\right)$. This is especially problematic for a low number of multi-skilled employees. In this case, the group staffing requirements need to be primarily satisfied by capacity buffers. However, the total capacity buffer that can be scheduled is limited by the total minimum staffing requirements $\left(R_{m d j}^{w}\right)$ and the time-related constraints. As such, it becomes difficult to appropriately position the capacity buffers, which is already identified by Ingels and Maenhout (2015).

\subsection{Robustness validation of employee substitutability}

In this section, we compare the resulting robustness for different proactive scheduling strategies applied to construct a personnel shift roster for the real-life staffing requirements. In this respect, we consider the average results over all hiring levels for the minimum cost roster without an increased employee substitutability ("minimum cost'), the personnel shift roster with group employee substitutability ('group substitutability') and a personnel shift roster with individual employee substitutability ('individual substitutability').

Figure 5 represents the variability of the expected actual cost corresponding to the different scheduling strategies and substitution types (cf. Table 6) for the real-life demand profile with the highest minimum staffing requirements. In this respect, we distinguish the best-case, average and worst-case expected actual cost and notice that considering any form of employee substitutability results in the lowest cost through a significantly lower number of shortages. Moreover, group employee substitutability is especially useful if only between-skill substitutions are considered.

We display the worst-case expected actual cost for the between-skill substitutions according to the different scheduling strategies in Fig. 6. This figure confirms the dominance of including some substitutability in the optimisation model over the minimum cost strategy for different degrees of cross-training of the workforce. Moreover, Fig. 6 indicates that the overall performance of the group substitutability actually stems from the beneficial impact of the created capacity buffers for a low number of multi-skilled employees in the tactical scheduling phase (cf. Fig. 4). As the number of multi-skilled employees rises, the individual employee substitutability strategy provides the best results. In order to compensate the poor performance of individual employee substitutability for a small number of multi-skilled employees, the value of within-skill and day-off-to-work substitutions should be optimised. In this respect, when all substitution types are considered, we notice that individual employee substitutability continuously outperforms group employee substitutability for all skill possession settings.

Regardless of the hiring level and the artificial and real-life demand profiles, employee substitutability on the level of the individual employee provides personnel shift rosters that exhibit a smaller variability and therefore a good average, best-case 


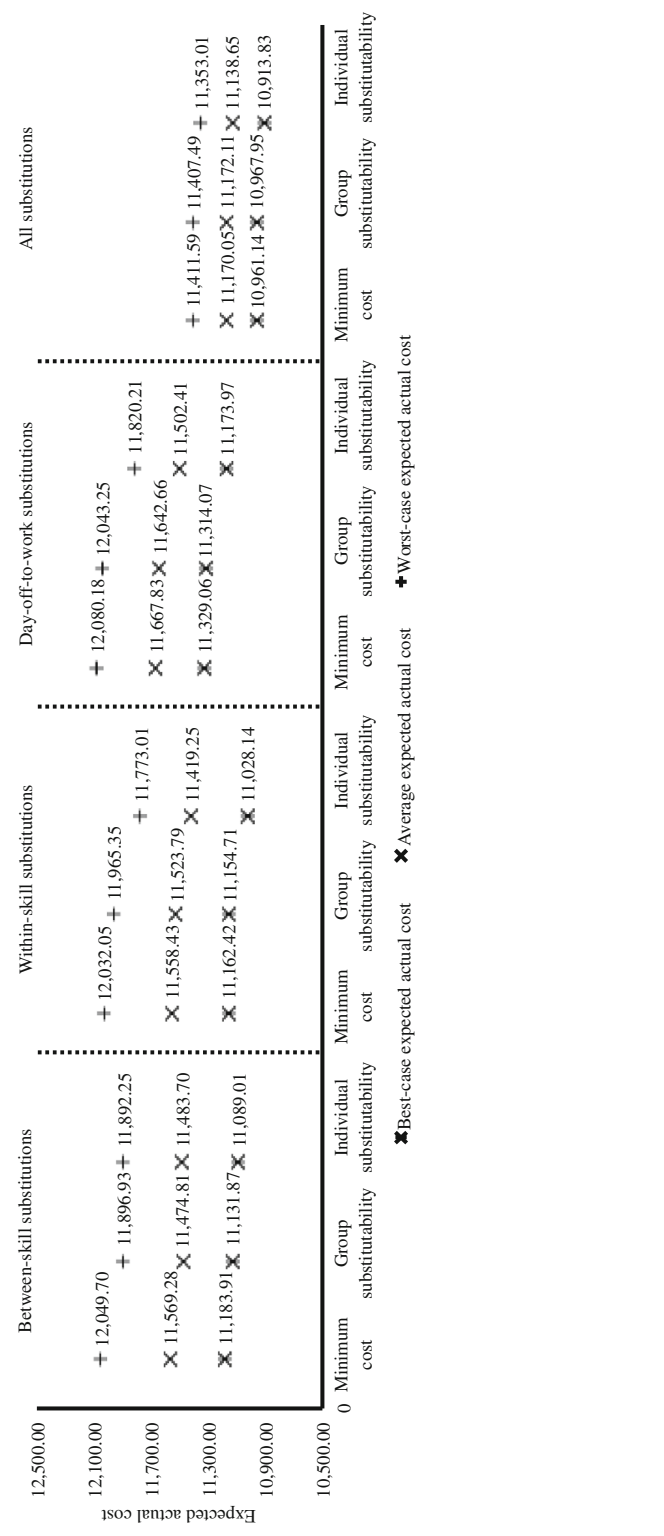

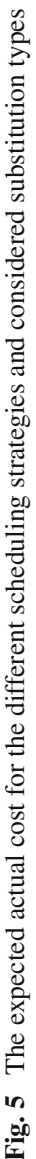




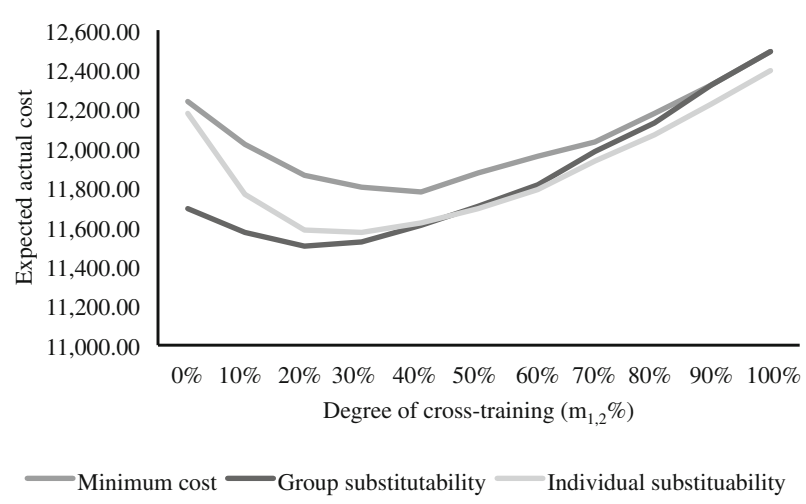

Fig. 6 The worst-case expected actual cost for the between-skill substitutions

and worst-case expected actual performance in the operational allocation phase. In fact, personnel shift rosters with individual employee substitutability are actually able to achieve a similar or better expected actual performance in terms of shortages with a smaller number of multi-skilled employees in comparison with the minimum cost personnel shift roster. In this respect, the personnel shift rosters with employee substitutability require less implicit flexibility in terms of multi-skilled employees to achieve the same or a higher flexibility. However, it is important to facilitate this flexibility in the operational allocation phase by limiting the change $\operatorname{cost}\left(c_{i m d j}^{w \delta}\right)$. Otherwise, the number of executed substitutions decreases and the individual employee substitutability strategy loses its value. In this case, personnel shift rosters with capacity buffers obtained by considering group employee substitutability provide a better actual performance.

\section{Conclusions}

In this paper, we consider a bi-objective personnel scheduling model that not only minimises the personnel assignment cost, but also maximises the employee substitutability to improve the robustness of a personnel shift roster. We define employee substitutability on the group level and on the level of the individual employee. Employee substitutability on the group level is characterised by group staffing requirements that determine the desired number of employees to be on duty able to carry out a specific skill. In order to maximise the individual employee substitutability of a tactical personnel shift roster, we consider three types of substitution possibilities between personnel members, i.e. between-skill, within-skill and day-off-to-work substitutions. Since we want to investigate the cost of robustness, we propose a two-phase pre-emptive programming approach.

The best strategy to improve the flexibility and the corresponding robustness of a tactical personnel shift roster is to define employee substitutability on the level of the individual employee. In the design of the proactive strategy as a proxy for the uncertainty and variability in the operational allocation phase, it is important to apply the correct strategy to count the number of substitution possibilities and to define the associated objective function structure in the tactical scheduling phase. 
The best strategy and objective function structure should depend on the variability of the minimum staffing requirements and the implicitly available flexibility, i.e. the number of multi-skilled employees and the considered substitution types, such that a sufficient but not excessive number of substitution possibilities is available and appropriately positioned. In this respect, the number of substitution possibilities that may be counted per duty should be limited to the minimum staffing requirements or to the number of scheduled duties in case of a small and large variability of the minimum staffing requirements, respectively. Moreover, a low implicit flexibility requires the definition of more additional substitution possibilities than a high implicit flexibility. This means that a low implicit flexibility requires a counting strategy that allows the definition of an unrestricted number of substitution possibilities per employee. In contrast, a higher implicit flexibility benefits from a more restrictive counting strategy. As such, the strategy should reflect the actual situation in the operational allocation phase where a single employee may only be reassigned to one other duty. In order to facilitate the availability and fitting positioning of these substitution possibilities, an appropriate cost of robustness and substitutability value should be defined. As more substitution possibilities need to be added and positioned, a higher cost increase or cost of robustness needs to be tolerated in the tactical scheduling phase. Furthermore, the position of the substitution possibilities should be carefully considered by determining the associated value based on the structure of the minimum staffing requirements.

Future research should focus on an expansion of the problem definition by considering hierarchical and fractional skills. Moreover, the planning horizon to adjust the baseline personnel shift roster in the operational allocation phase can be expanded to enable the reassignment of all future assignments.

Acknowledgements We acknowledge the support for the doctoral research project fundings by the Bijzonder Onderzoekfonds (BOF, Ghent University) under contract number 01N00712 and the National Bank of Belgium.

\section{Appendix: Operational allocation model}

\section{Notation}

Sets

$G \quad$ set of skills (index $m$ )

$N \quad$ set of employees (index $i$ )

$S \quad$ set of shifts (index $j$ )

General parameters

$\begin{array}{ll}d & \text { day under consideration in the operational planning horizon } \\ b_{i m} & 1 \text { if employee } i \text { possesses skill } m, 0 \text { otherwise } \\ c_{i m d j}^{w} & \text { wage cost of assigning an employee } i \text { to shift } j, \text { day } d \text { and skill } m \\ c_{m d j}^{w u} & \text { shortage cost for shift } j, \text { day } d \text { and skill } m \\ p_{i d j} & \text { preference penalty cost if an employee } i \text { receives a shift assignment } j \\ & \text { on day } d \\ l_{j} & \text { duration of shift } j\end{array}$


$\kappa_{i d j}^{\alpha} \quad 1$ if employee $i$ is allowed to receive an assignment during shift $j$ on day $d, 0$ otherwise

$\kappa_{i d}^{f} \quad$ the total number of hours employee $i$ has to receive on day $d$

Simulation parameters

$\begin{array}{ll}a_{i d} & 1 \text { if employee } i \text { is available on day } d, 0 \text { otherwise } \\ R_{m d j}^{\prime w} & \text { simulated staffing requirements for shift } j, \text { day } d \text { and skill } m\end{array}$

Roster change parameters

$x_{i m d j}^{\prime} w$

1 if employee $i$ received a shift assignment $j$ for skill $m$ on day $d$ in the baseline personnel shift roster, 0 otherwise

$c_{i m d j}^{w \delta}$

roster change cost for assigning an employee $i$ to shift $j$, day $d$ and skill $m$ with $c_{i m d j}^{w \delta}>0$ if $x_{i m d j}^{\prime w}=0 c_{i m d j}^{w \delta}=0$ otherwise

$c_{i d}^{v} \quad$ duty cancellation cost for employee $i$ on day $d$ with $c_{i d}^{v}>0$ if $\sum_{m \in G} \sum_{j \in S} x_{i m d j}^{\prime w}=1$ and $a_{i d}=1 \quad c_{i d}^{v}=0$ otherwise

Variables

$x_{i m d j}^{w} \quad 1$ if employee $i$ receives a shift assignment $j$ for skill $m$ on day $d, 0$ otherwise

$x_{i d}^{v} \quad 1$ if employee $i$ receives a day off on day $d, 0$ otherwise

$x_{m d j}^{w u}$ the shortage of employees for shift $j$, day $d$ and skill $m$

\section{Mathematical formulation}

$$
\begin{aligned}
& \min \sum_{i \in N} \sum_{m \in G} \sum_{j \in S}\left(c_{i m d j}^{w}+c_{i m d j}^{w \delta}+p_{i d j}\right) x_{i m d j}^{w}+\sum_{i \in N} c_{i d}^{v} x_{i d}^{v} \\
& +\sum_{m \in G} \sum_{j \in S} c_{m d j}^{w u} x_{m d j}^{w u} \\
& \sum_{i \in N} b_{i m} x_{i m d j}^{w}+x_{m d j}^{w u} \geq R_{m d j}^{\prime w} \quad \forall m \in G, \forall j \in S \\
& \sum_{m \in G} \sum_{j \in S} x_{i m d j}^{w} \leq a_{i d} \quad \forall i \in N \\
& \sum_{m \in G} \sum_{j \in S} x_{i m d j}^{w}+x_{i d}^{v}=1 \quad \forall i \in N \\
& \sum_{m \in G} x_{i m d j}^{w} \leq \kappa_{i d j}^{\alpha} \quad \forall i \in N, \forall j \in S \\
& \sum_{m \in G} \sum_{j \in S} l_{j} x_{i m d j}^{w} \geq \kappa_{i d}^{f} a_{i d} \quad \forall i \in N \\
& x_{i m d j}^{w} \in\{0,1\} \quad \forall i \in N, \forall m \in G, \forall j \in S \\
& x_{i d}^{v} \in\{0,1\} \quad \forall i \in N \\
& x_{m d j}^{w u} \geq 0 \quad \forall m \in G, \forall j \in S \\
& \forall m \in
\end{aligned}
$$


The objective function (Eq. 24) minimises the wage cost, roster change cost, preference penalty cost, cancellation cost and the cost for understaffing. The objective function weights are as follows, i.e.

- Every employee has a wage cost $\left(c_{i m d j}^{w}\right)$ of $10 \times 1.2^{\sum_{m \in G} b_{i m}-1}$.

- The roster change cost $\left(c_{i m d j}^{w \delta}\right)$ depends on the chosen scenario (cf. Table 6).

- Every employee has a preference penalty cost $\left(p_{i d j}\right)$ that is randomly generated in the range of 1 to 5 .

- The duty cancellation cost $\left(c_{i d}^{v}\right)$ is 5 .

- The shortage cost $\left(c_{m d j}^{w u}\right)$ is fixed at 20.

Constraint (25) imposes the staffing requirements and every employee can only receive a shift assignment if (s)he is available (Eq. 26). Constraint (27) ensures that every employee receives either a shift assignment or a day off. The shifts assigned to the employees need to satisfy the time-related constraints (Eqs. 4-7). The satisfaction of the minimum rest period (Eq. 4), maximum number of hours that can be assigned (Eq. 5) and maximum consecutive working assignments (Eq. 7) is ensured through the definition of $\kappa_{i d j}^{\alpha}$ in constraint (28). Finally, constraint (29) ensures that every employee works a minimum number of hours over the complete planning period (Eq. $6)$. Note that if an employee is unavailable on a working day $\left(a_{i d}=0\right)$, we adapt the minimum number of hours for this employee such that the employee does not have to catch up this duty. We define the integrality conditions in Eq. (30).

\section{References}

Abdelghany A, Ekollu G, Narasimhan R, Abdelghany K (2004) A proactive crew recovery decision support tool for commercial airlines during irregular operations. Ann Oper Res 127(1-4):309-331

Abdelghany K, Abdelghany A, Ekollu G (2008) An integrated decision support tool for airlines schedule recovery during irregular operations. Eur J Oper Res 185(2):825-848

Abernathy W, Baloff N, Hershey J (1973) A three-stage manpower planning and scheduling model a service sector example. Oper Res 21:693-711

Ahmed M, Alkhamis T (2009) Simulation optimization for an emergency department healthcare unit in Kuwait. Eur J Oper Res 198(3):936-942

Bailyn L, Collins R, Song Y (2007) Self-scheduling for hospital nurses: an attempt and its difficulties. J Nurs Manag 15(1):72-77. doi:10.1111/j.1365-2934.2006.00633.x

Bard J, Purnomo H (2005) Hospital-wide reactive scheduling of nurses with preference considerations. IIE Trans 37:589-608

Bard J, Purnomo H (2005) Preference scheduling for nurses using column generation. Eur J Oper Res 164:510-534

Bard J, Purnomo H (2005) Short-term nurse scheduling in response to daily fluctuations in supply and demand. Health Care Manag Sci 8:315-324

Bertsimas D, Sim M (2004) The price of robustness. Oper Res 52(1):35-53. http://www.jstor.org/stable/ 30036559

Bertsimas D, Thiele A (2006) A robust optimization approach to inventory theory. Oper Res 54(1):150-168. doi:10.1287/opre.1050.0238

Brucker P, Burke EK, Curtois T, Qu R, Vanden Berghe G (2010) A shift sequence based approach for nurse scheduling and a new benchmark dataset. J Heuristics 16(4):559-573. doi:10.1007/ s10732-008-9099-6

Bureau of Labor Statistics: Absences from work of employed full-time wage and salary workers by occupation and industry (table 47). http://www.bls.gov/cps/cpsaat47.htm (2013)

Burke E, De Causmaecker P, Vanden Berghe G, Van Landeghem H (2004) The state of the art of nurse rostering. J Sched 7:441-499 
Campbell G (1999) Cross-utilization of workers whose capabilities differ. Manag Sci 45:722-732

Davenport A, Gefflot C, Beck C (2001) Slack-based techniques for robust schedules. In: Sixth European conference on planning

De Bruecker P, Van den Bergh J, Beliën J, Demeulemeester E (2015) Workforce planning incorporating skills: state of the art. Eur J Oper Res 243(1):1-16. doi:10.1016/j.ejor.2014.10.038, http://www. sciencedirect.com/science/article/pii/S0377221714008601

De Causmaecker P, Vanden Berghe G (2003) Relaxation of coverage constraints in hospital personnel rostering. Lecture notes in computer science 2740

De Causmaecker P, Vanden Berghe G (2011) A categorisation of nurse rostering problems. J Sched 14:3-16

Dillon J, Kontogiorgis S (1999) US Airways optimizes the scheduling of reserve flight crews. Interfaces 29(5):95-122

Dorne R (2008) Personnel shift scheduling and rostering. In: Voudouris C, Lesaint D, Owusu G (eds) Service chain management. Springer, Berlin, pp 125-138. doi:10.1007/978-3-540-75504-3_9

Dowsland K, Thompson J (2000) Solving a nurse scheduling problem with knapsacks, networks and tabu search. J Oper Res Soc 51:825-833

Dück V, Ionescu L, Kliewer N, Suhl L (2012) Increasing stability of crew and aircraft schedules. Transp Res Part C Emerg Technol 20(1):47-61

Eggenberg N, Salani M, Bierlaire M (2010) Constraint-specific recovery network for solving airline recovery problems. Comput Oper Res 37(6):1014-1026. doi:10.1016/j.cor.2009.08.006, http://www. sciencedirect.com/science/article/pii/S030505480900210X

Ehrgott M, Ryan DM (2002) Constructing robust crew schedules with bicriteria optimization. J MultiCriteria Decis Anal 11(3):139-150

Ernst A, Jiang H, Krishnamoorthy M, Owens B, Sier D (2004a) An annotated bibliography of personnel scheduling and rostering. Ann Oper Res 127:21-144

Ernst A, Jiang H, Krishnamoorthy M, Sier D (2004b) Staff scheduling and rostering: a review of applications, methods and models. Eur J Oper Res 153:3-27

European Foundation for the Improvement of Living and Working Conditions: absence from work: executive summary. http://eurofound.europa.eu/observatories/eurwork/comparative-information/ absence-from-work (2010)

Gao C, Johnson E, Smith B (2009) Integrated airline fleet and crew robust planning. Transp Sci 43(1):2-16. doi: $10.1287 / \operatorname{trsc} .1080 .0257$

Gross CN, Fügener A, Brunner JO (2017) Online rescheduling of physicians in hospitals. Flex Serv Manuf J. doi:10.1007/s10696-016-9274-2

Gurobi Optimization I (2014) Gurobi optimizer reference manual (2014). http://www.gurobi.com

Hazir O, Haouari M, Erel E (2010) Robust scheduling and robustness measures for the discrete time/cost trade-off problem. Eur J Oper Res 207:633-643

Ikegami A, Niwa A (2003) A subproblem-centric model and approach to the nurse scheduling problem. Math Program 97:517-541

Ingels J, Maenhout B (2015) The impact of reserve duties on the robustness of a personnel shift roster: An empirical investigation. Comput Oper Res 61:153-169. doi:10.1016/j.cor.2015.03.010

Ionescu L, Kliewer N (2011) Increasing flexibility of airline crew schedules. Procedia Soc Behav Sci 20:1019-1028

Maenhout B, Vanhoucke M (2010) Branching strategies in a branch-and-price approach for a multiple objective nurse scheduling problem. J Sched 13:77-93

Moudani W, Mora-Camino F (2010) Solving crew reserve in airlines using dynamic programming approach. Int J Optim Theory Methods Appl 2(4):302-329

Olivella J, Nembhard D (2016) Calibrating cross-training to meet demand mix variation and employee absence. Eur J Oper Res 248(2):462-472. doi:10.1016/j.ejor.2015.07.036

Pato M, Moz M (2008) Solving a bi-objective nurse rerostering problem by using a utopic Pareto genetic heuristic. J Heuristics 14:359-374

Potthoff D, Huisman D, Desaulniers G (2010) Column generation with dynamic duty selection for railway crew rescheduling. Transp Sci 44(4):493-505

Rosenberger J, Schaefer A, Goldsman D, Johnson E, Kleywegt A, Nemhauser G (2002) A stochastic model of airline operations. Transp Sci 36(4):357-377

SD Worx: out of office: Ziekteverzuim in België 2012 (2013)

Shebalov J, Klabjan D (2006) Robust airline crew pairing: move-up crews. Transp Sci 40:300-312

Sohoni M, Johnson E, Bailey T (2006) Operational airline reserve crew planning. J Sched 9(3):203-221 
Soyster AL (1973) Convex programming with set-inclusive constraints and applications to inexact linear programming. Oper Res 21(5):1154-1157. http://www.jstor.org/stable/168933

Tam B, Ehrgott M, Ryan DM, Zakeri G (2011) A comparison of stochastic programming and bi-objective optimisation approaches to robust airline crew scheduling. OR Spectr 33(1):49-75

Tam B, Ryan D, Ehrgott M (2014) Multi-objective approaches to the unit crewing problem in airline crew scheduling. J Multi-Criteria Decis Anal 21(5-6):257-277. doi:10.1002/mcda.1517

Topaloglu S, Ozkarahan I (2004) An implicit goal programming model for the tour scheduling problem considering the employee work preferences. Ann Oper Res 128(1):135-158. doi:10.1023/B:ANOR. 0000019102.68222.df

Topaloglu S, Selim H (2010) Nurse scheduling using fuzzy modelling approach. Fuzzy Sets Syst 161:15431563

Trivedi V, Warner D (1976) A branch and bound algorithm for optimum allocation of float nurses. Manag Sci 22:972-981

Van den Bergh J, Beliën J, De Bruecker P, Demeulemeester E, De Boeck L (2013) Personnel scheduling: a literature review. Eur J Oper Res 226:367-385

Vanhoucke M, Maenhout B (2009) On the characterization and generation of nurse scheduling problem instances. Eur J Oper Res 196:457-467

Yeh JY, Lin WS (2007) Using simulation technique and genetic algorithm to improve the quality care of a hospital emergency department. Expert Syst Appl 32(4):1073-1083 